\title{
Alternative splicing liberates a cryptic cytoplasmic isoform of mitochondrial MECR that antagonizes influenza virus
}

\author{
Steven F. Baker ${ }^{1}$, Helene Meistermann², Manuel Tzouros², Aaron Baker ${ }^{3,4}$, Sabrina Golling ${ }^{2}$, \\ Juliane Siebourg Polster², Mitchell P. Ledwith", Anthony Gitter ${ }^{3,4,5}$, Angelique Augustin², \\ Hassan Javanbakht6 ${ }^{6,}$, Andrew Mehle ${ }^{1,7}$
}

\begin{abstract}
Viruses must balance their reliance on host cell machinery for replication while avoiding host defense. They often exploit conserved essential host genes whose critical role for the cell limits mutational escape. Conversely, host antiviral genes are often nonessential and can undergo mutation or regulated expression to thwart infection and limit self damage. Influenza $A$ viruses are zoonotic agents that frequently switch hosts, causing localized outbreaks with the potential for larger pandemics. The host range of influenza virus is limited by the need for successful interactions between the virus and cellular partners. Here we used immuno-competitive capturemass spectrometry to identify cellular proteins that interact with human- and avian-style viral polymerases. We focused on the pro-viral activity of heterogenous nuclear ribonuclear protein Ulike 1 (hnRNP UL1) and the anti-viral activity of mitochondrial enoyl CoA-reductase (MECR). MECR is localized to mitochondria where it functions in mitochondrial fatty acid synthesis (mtFAS). While a small fraction of the polymerase subunit PB2 localizes to the mitochondria, we could not confirm interactions with full-length MECR. By contrast, RNA-seq revealed a minor splice variant that creates cytoplasmic MECR (CMECR). CMECR engages the viral polymerase and suppresses viral replication. MECR ablation through genome editing or drug treatment is detrimental for cell health, creating a generic block virus replication. Using the yeast homolog Etr1 to supply the metabolic functions of MECR, we showed that specific antiviral activity is independent of mtFAS and lies solely within cMECR. Thus, a cryptic antiviral activity is embedded within a key metabolic enzyme, possibly protecting it from viral countermeasures.
\end{abstract}

\section{Introduction}

Yearly influenza virus epidemics shape public health programs worldwide. Protection through vaccination and treatment with antivirals helps slow influenza virus, yet infections still cause $\sim 61,000$ deaths yearly in the United States during high severity seasons (Garten et al., 2018). Migratory waterfowl are the natural host reservoir for influenza A viruses. Spillover into new hosts and adaptation has led to endemic infection in mammals including humans, pigs, dogs, and horses. To move from one host to the next, viruses must overcome cross-species transmission barriers by engaging divergent cellular co-factors while evading restriction factors. Emerging and reemerging influenza viruses regularly surmount host barriers through adaptive mutations that allow them to interface with divergent host cell environments. Thus, it is paramount to understand what conserved cellular functions are engaged by viruses and allow them to establish infection, especially during initial cross-species events.

Viruses are completely dependent upon cellular co-factors. This dependence can be countered by hosts through positive selection of escape mutations on critical co-factors or antiviral proteins. The recursive process of host evasion

\footnotetext{
${ }^{1}$ Department of Medical Microbiology and Immunology, University of Wisconsin-Madison, USA. ${ }^{2}$ Roche Pharma Research and Early Development, Pharmaceutical Sciences - Biomarkers, Bioinformatics and Omics \& Pathology, Roche Innovation Center Basel, F. HoffmannLa Roche Ltd, Basel, Switzerland. ${ }^{3}$ Department of Computer Sciences, University of Wisconsin-Madison. ${ }^{4}$ Morgridge Institute for Research, Madison, Wisconsin. ${ }^{5}$ Department of Biostatistics and Medical Informatics, University of Wisconsin-Madison. ${ }^{6}$ Roche Pharma Research and Early Development, Infectious Diseases, Roche Innovation Center Basel, F. Hoffmann-La Roche Ltd, Basel, Switzerland. * current affiliation: SQZ Biotechnologies, 200 Arsenal Yards Blvd, Suite 210, Watertown, MA 02472. ${ }^{7}$ Corresponding author: amehle@wisc.edu @mehlelab
} 
countered by viral adaptation establishes a socalled molecular arms race, or Red Queen genetic conflict (Van Valen, 1976). The process is aided by the fact that most host antiviral genes are nonessential for the host cell, enabling mutation without compromising viability, and mutational tolerance is further bolstered through gene duplication and transcriptional regulation (Daugherty and Malik, 2012). Gene duplication and diversification allows hosts to mutate otherwise essential genes whereas changes in transcriptional regulation can selectively activate genes should their constitutive expression be detrimental. To counter mutational tolerance, it has been suggested that viruses target essential genes as host co-factors, because the genes are less prone to variability (Rialdi et al., 2017).

Due to their rapid replication and high mutation rates relative to the host, viruses are eventually successful in both winning genetic conflicts and adapting to new hosts. For example, the human protein ANP32A or its paralog ANP32B are required for influenza viral genome replication (Sugiyama et al., 2015). While ANP32A/B double knockout mice are not viable, functional overlap between the cellular requirements of both proteins theoretically allows hosts to test virus escape mutations without a strong fitness cost (Reilly et al., 2011). This is perhaps exemplified by the insertion of duplicated sequence in the avian ANP32A locus and loss-of-function mutation in $A N P 32 B$. This has forced avian-adapted influenza polymerases to adapt where they have become solely reliant upon avian ANP32A (Long et al., 2016, 2019). As a consequence, avian-adapted viral polymerase function poorly in mammals (Almond, 1977). Nonetheless, a single amino acid change caused by a single nucleotide mutation in the viral polymerase PB2 subunit (E627K) allows avian influenza viruses to rapidly adapt to and exploit human ANP32A (Subbarao et al., 1993).

The viral ribonucleoprotein (VRNP) is the minimal unit for viral genome replication and a major hotspot for influenza virus host-adaptation. RNPs are helically wound structures composed of genomic RNA encapsidated by NP with the heterotrimeric RNA-dependent RNA polymerase at one end binding both the $5^{\prime}$ and 3 ' termini of the genome. The polymerase is composed of the PB1, PB2 and PA subunits. All of the enzymatic activities required for replication and transcription are intrinsic to the polymerase, whereas host factors serve as essential co-factors or modulate vRNP function (Dawson et al., 2020; Peacock et al.,
2019). The viral polymerase replicates the minussense genomic vRNA into a plus-sense cRNA intermediate, which is then copied back to vRNA. The polymerase also transcribes viral mRNA from the vRNA template. The polymerase assumes distinct conformations during each step, presenting unique interfaces for host protein interactions (reviewed in (Fodor and te Velthuis, 2019; Wandzik et al., 2020)). Indeed, MCM, ANP32A or ANP32B, and RNAP2 are host proteins that facilitate cRNA synthesis, vRNA synthesis, and transcription, respectively (Engelhardt et al., 2005; Kawaguchi and Nagata, 2007; Sugiyama et al., 2015).

Understanding which host proteins the polymerase requires for replication, especially those that it engages when influenza virus jumps from one host to the next, is essential for understanding the genetic conflicts that establish barriers to crossspecies transmission and shape viral evolution. Transmission of influenza virus from birds to humans requires that the incoming vRNPs successfully interact with cellular factors to, at a minimum, provide sufficient levels of replication during which adaptive mutations can arise. To understand how avian viruses engage the foreign intracellular environment of human cells, we used immuno-competitive capture-mass spectrometry (ICC-MS) to define interaction networks between human proteins and avian or human-adapted viral PB2. We identified heterogenous nuclear ribonuclear protein U-like 1 (hnRNP UL1) as a cellular co-factor that supports influenza virus replication and mitochondrial enoyl CoA-reductase (MECR) that plays an anti-viral role. MECR localizes to the mitochondria and is a critical enzyme in mitochondrial fatty acid synthesis (mtFAS), raising questions as to how it could counteract the viral polymerase in the cell nucleus. Surprisingly, we demonstrated that MECR antiviral activity is derived from a cryptic splice isoform that produces cytosolic MECR (cMECR). Loss of MECR cripples mtFAS and cellular metabolism, resulting in defects in cell health and a generic block to viral replication. However, repairing MECR deficient cells with the yeast homolog Etr1, which lacks a cMECR-like isoform, showed that CMECR alone suppresses influenza virus replication independent of mtFAS. Thus, MECR conceals the antiviral protein CMECR that is revealed by differential splicing. Our findings suggest that burying antiviral function within an essential host gene potentially safeguards the host from viral antagonism. 
a

FLAG-tagged influenza virus infection

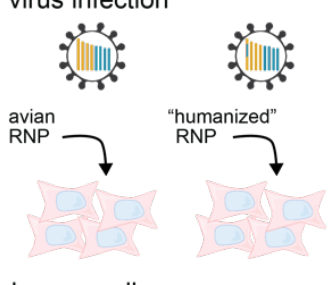

human cells

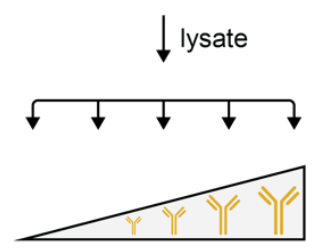

competing anti-FLAG $A b$
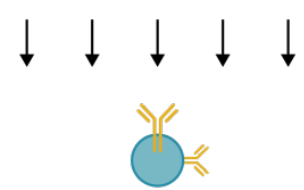

immobilized anti-FLAG immunoprecipitation

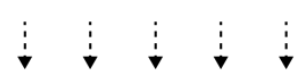

LC-MS/MS
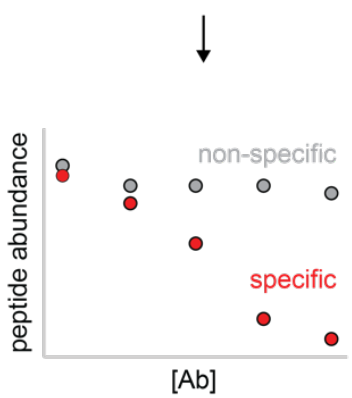

[Ab] b

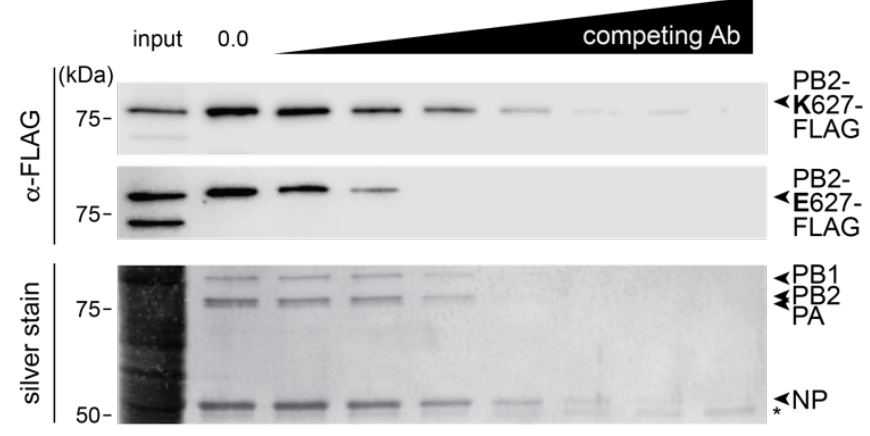

d Protein-protein interaction modules

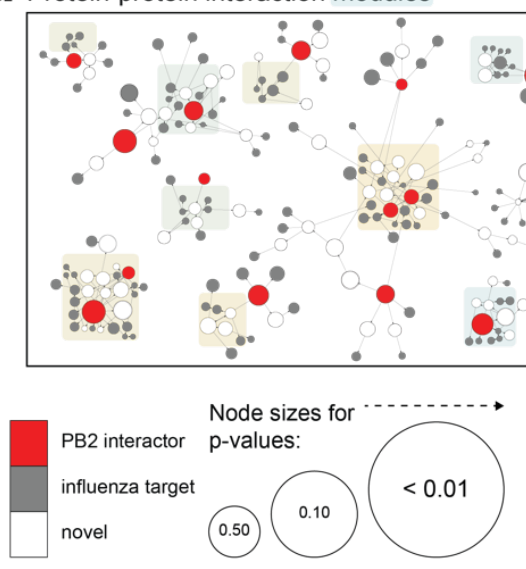

Top GO term per module

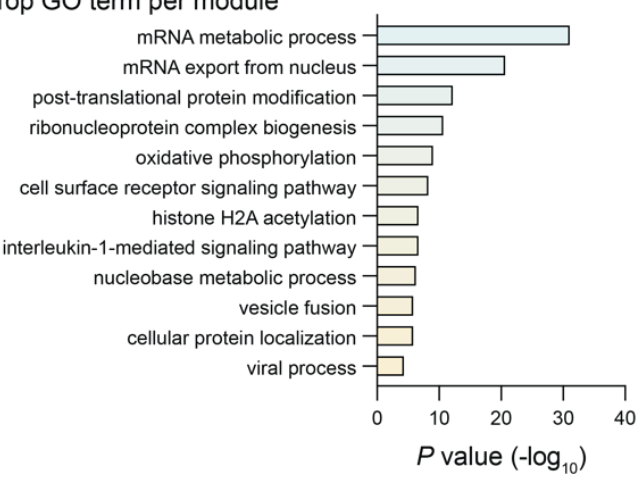

$P$ value $\left(-\log _{10}\right)$
C

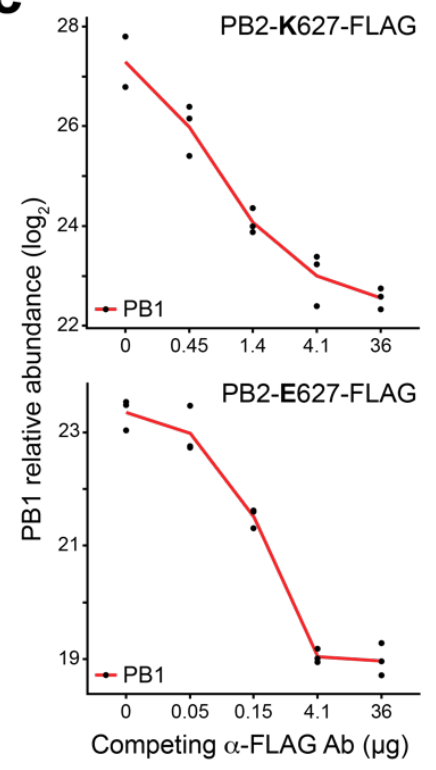

Fig. 1: ICC-MS defines an influenza polymerase interactome. a, Schematic diagram to identify PB2 interactome. Lysate fractions from human A549 cells infected with PB2FLAG tagged influenza virus encoding avian RNP or the "humanized" PB2-E627K RNP were incubated with competing soluble anti-FLAG antibody followed by capture with resin-bound anti-FLAG and LC-MS/MS. b-c, Immuno-competitive capture of the viral RNP. b. Detection of PB2 627E and 627K (top: western blot) or PB2 627K with coprecipitating RNP components (bottom: silver stain). ${ }^{*}$, IgG heavy chain. c, Relative protein abundance of PB1 in PB2 ICC-MS samples shows decreasing capture with increasing competition antibody. Data shown are in biological triplicate. d, Minimum-cost flow simulations connect top PB2 interactors identified by ICC-MS (red) to previously identified influenza host factors (gray), in some cases through other host proteins (white). Modules comprising different PB2 interactors were enriched for GO terms, the most significant of which is enlarged. Node sizes indicate empirical $\mathrm{P}$ values derived from the control flow simulations.

\section{Results}

Identification of host proteins and pathways that interface with influenza virus polymerase.

During zoonoses, avian influenza virus polymerases must co-opt mammalian host processes and proteins to direct virus replication. To identify polymerase co-factors important for zoonotic and endemic transmission, we infected human lung cells with virus encoding avian-style PB2 E627 or the human style PB2 K627 and performed affinity purification-MS (AP-MS) for
PB2. As these experiments were performed using infected cells, PB2 exists alone, as part of the heterotrimeric polymerase, or as part of the larger RNP. AP-MS approaches can be complicated by high levels of nonspecific interactions. We overcame these limitations by performing ICC-MS (Fig. 1a). ICC-MS is a label-free strategy that includes a competition step with in-solution antibody prior to affinity purification using antibody already bound to a solid support. ICC-MS thus distinguishes specific interactions, which can be 
competed, from nonspecific interactions, which are unaffected by the competition step (Meistermann et al., 2014). The competition profiles are further used to rank-order putative host interactors. Increasing amounts of competing antibody specifically reduced capture of $\mathrm{PB} 2$ and the viral RNP (Fig. 1b). Avian-style PB2 E627 was more sensitive to competition, possibly because this virus is restricted in human cells and expresses lower levels of viral proteins (Mehle and Doudna, 2009). The samples were then processed for protein identification by LC-MS/MS. Effective competition was confirmed where increased competitor antibody decreased the relative abundance of PB2 itself, as well as the RNP components PB1, PA, and NP that interact with PB2 (Fig. 1c and Supp. Fig. 1a). 1,377 proteins were identified in precipitates from PB2 K627 or E627 infections at all five antibody concentrations and in at least two of three biological replicates. Hits were prioritized based on their competition curves profile to produce a focused list of 22 candidates (Supp. Table 1). We also included ANP32A, EWSR1, FUS, and GMPS that were identified in a pilot screen, but not among the top candidates in the subsequent analysis. Many of the PB2 interactors were previously identified in proteomic screens for influenza virus polymerase co-factors and the ICC-MS hits ADAR, ANP32A, ATP7A, and KPNA3 were studied in detail, providing confidence in our approach (de Chassey et al., 2013; Gabriel et al., 2011; Rupp et al., 2017; Sugiyama et al., 2015). The majority of PB2 interactors were found during infection with avianor human-style polymerases, suggesting the identification of co-factors conserved for both zoonotic and endemic infections.

To increase the power of our ICC-MS results, we performed protein-protein interaction network analyses tailored for influenza virus. Networks were constructed of experimentally demonstrated protein-protein interactions (STRING; (Szklarczyk et al., 2015)). We then performed minimum-cost flow simulations where the candidate PB2 interactors served as sources to link to targets marked as influenza host factors based on data from six genome-wide screens (Brass et al., 2009; Hao et al., 2008; Karlas et al., 2010; König et al., 2010; Larson et al., 2019; Shapira et al., 2009; Tran et al., 2020). 23 of the 26 PB2 interactors identified by ICC-MS readily formed subnetworks with previously identified influenza host factors and revealed key cellular processes defined by GO enrichment scores for each subnetwork (Fig. 1d; Supp. Table 2). Further, they uncovered new protein partners within these subnetworks and connected to highly significant modules not previously associated with influenza virus

Flow simulations can be biased due to nodes forming spurious links in order to reach large multi-partner targets. We performed two separate minimum-cost flow simulation experiments to control for this possibility. In one, we used the same known influenza host factors as targets but replaced the PB2 interactors as source proteins with proteins randomly sampled from STRING. These results demonstrated that most of the flow subnetworks containing ICC-MS hits were specific to those generated by PB2 interactors and not randomly sampled proteins (Fig. 1d; Supp. Fig. 1b,c; Supp. Table 2a). In the second control experiment, we performed minimum-cost flow simulations for a different virus, hepatitis $C$ virus (HCV). Host proteins that interact with the HCV NS5A protein were used as sources to link to targets identified by a genome-wide screen for HCV host factors (Supp. Table 2b). The simulation demonstrated that the connection of most PB2 interactors to important viral cofactor nodes was specific to the influenza virus-defined network, and not the HCV-defined network. Both controls confirm that our PB2 interactors connect to subnetworks relevant for influenza virus, and not general virus replication or generic hubs. To further test the networks, we queried them using host proteins with thoroughly studied interactions as sources: PKR and RIG-I, proteins important for innate immune defense against influenza virus (reviewed in (García et al., 2006; Rehwinkel and Gack, 2020)); CRM1 and NXF1, proteins important for viral nuclear-cytoplasmic transport (Neumann et al., 2000; Satterly et al., 2007); and, EXOSC3 and UBR4, proteins that were identified through rigorous omics approaches (Rialdi et al., 2017; Tripathi et al., 2015). These six proteins were used as sources to connect to influenza host factor targets, then controlled through two separate simulations as above. Both flow simulations produced similar results that recapitulate in silico the biochemically-defined interaction networks, and suggest new proteins that may be important for these processes (Supp. Fig. 1d; Supp. Table 2c,d).

The influenza networks were used to interrogate PB2 interactors. The interactors formed twelve distinct modules, each enriched for different cellular processes. For example, mRNA metabolic 
process was the most significantly enriched GO term associated with the module containing PB2 ICC-MS hits HNRNPUL1, FUS, and SRSF2, 21 other viral co-factors, and five proteins not previously implicated during infection (Fig. 1d, Supp. Table 2e,f). Of the 23 mapped interactors, seven (ALDH18A1, FUS, GPD2, KPNA4, MRTO4, SRSF2, and TRMT61A, SRSF2) did not share direct edges with host co-factors but did connect to co-factors via an intermediary protein that has not been previously implicated. Together, these results provide confidence in the specificity and biological relevance of the ICC-MS hits and suggest additional host proteins and pathways that may be important for polymerase function.

The host protein hnRNP UL1 promotes and MECR restricts viral infection. To test the functional role of proteins identified by ICC-MS, candidate interactors were knocked down by siRNA treatment in A549 cells prior to infection with influenza virus (Fig. 2a). Infections were performed with human-style PB2 K627 and avian-style E627 viruses to detect any species-specific dependence. NXF1, an essential host co-factor, was knocked down as a positive control and caused a severe decrease in replication, as expected (Hao et al., 2008). Knockdown of hnRNP UL1 reduced viral titers to $\sim 25 \%$ of the non-targeting control, whereas knockdown of MECR significantly increased titers 2.5 to 5 -fold. Knockdown of other interactors had only modest effects in A549 cells, possibly because these factors function redundantly (e.g. importin- $\alpha$ isoforms, ANP32A and ANP32B), are needed in very limited quantities (e.g. ANP32A), or are not essential for viral replication (Baker et al., 2018; Gabriel et al., 2011). Titers in the knockdown cells for virus encoding PB2 K627 or PB2 E627 were highly correlated (Pearson's $r^{2}=0.791$ ), suggesting our interactors had comparable roles for human-signature or avian-signature virus polymerases (Fig. 2b).

$$
\text { Separate experiments confirmed }
$$
knockdown of hnRNP UL1 and MECR (Fig. 2c). Reductions in hnRNP UL1 protein levels decreased viral replication, whereas reduction in MECR protein levels increased titers. Similar knockdown phenotypes were detected during multicycle replication in another human cell line, 293T (Supp. Fig. 2a,b). We extended these findings to primary isolates of influenza $A$ virus from the 2009 pandemic (A/California/04/2009 [CA04]; H1N1) and influenza $B$ virus (B/Brisbane/60/2008 [B/Bris]) (Fig. 2d). Loss of
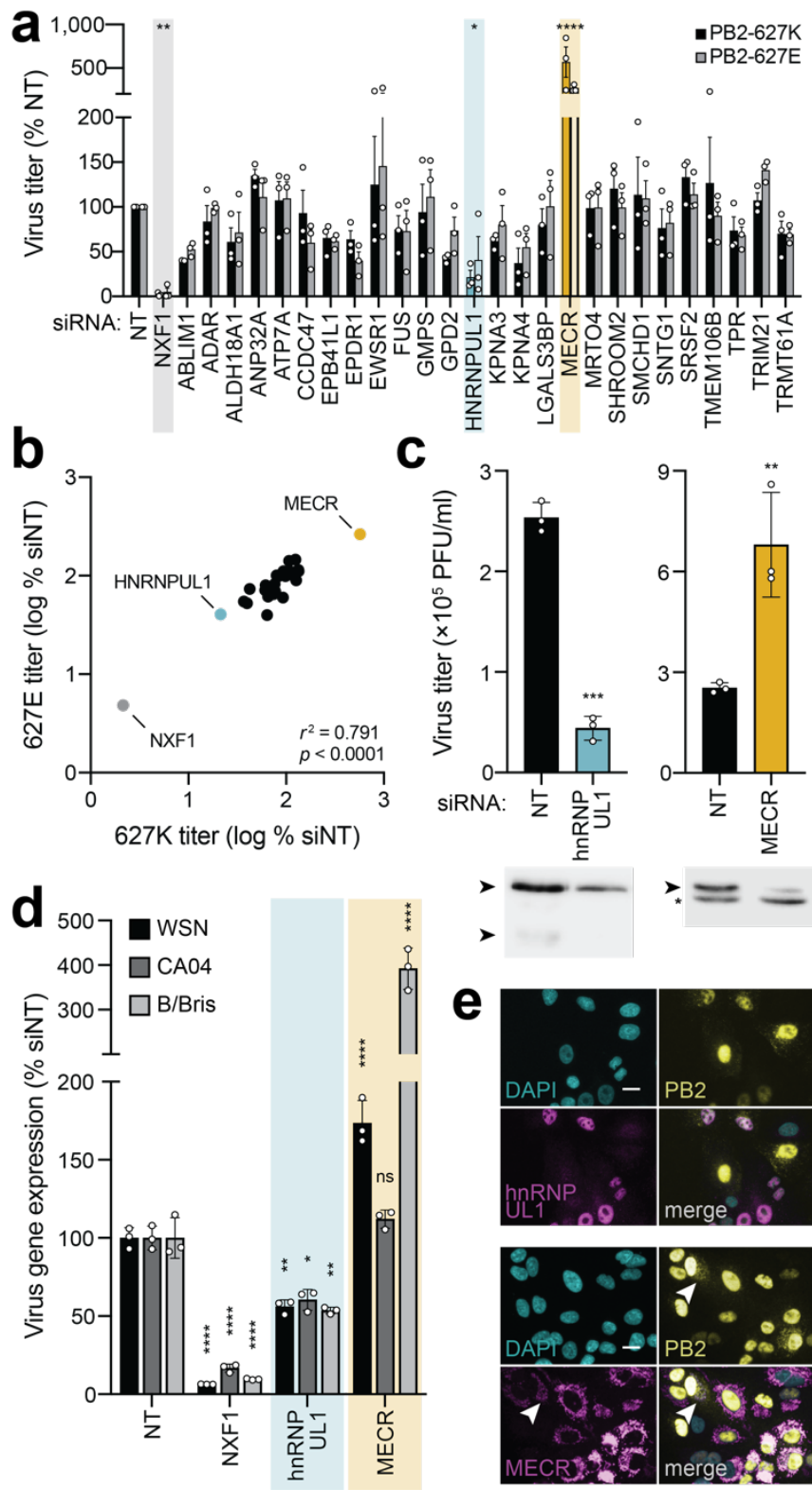

Fig. 2: Functional analysis of top candidate PB2 interactors reveals important roles for hnRNP UL1 and MECR. a, Secondary screening of proteomic hits by siRNA treatment and reporter virus infection. After knockdown, A549 cells were infected with human (PB2-627K; MOI, 0.01) or avian-adapted (PB2-627E; MOI, 0.05) WSN NLuc virus for $24 \mathrm{~h}$. Viral supernatants were titered and normalized to a non-targeting control (NT). Control NXF1 (gray) and outliers highlighted (hnRNP UL1, cyan; MECR, yellow). b, Concordance of virus titer for PB2-627E vs PB2-627K virus infections in siRNAtreated cells (from a). Statistical analysis performed with a two-tailed Pearson correlation coefficient. c, Multi-cycle virus replication of WT virus was measured $24 \mathrm{~h}$ post infection in A549 cells treated with the indicated siRNAs. Knockdown efficiency was analyzed by western blot. Asterisk indicates non-specific band. d, Knockdown impacts viral gene expression of divergent influenza viruses. siRNA-treated A549 cells were infected with reporter viruses based on WSN (pre-2009 H1N1; MOI, 0.1), CA04 (pandemic $2009 \mathrm{H} 1 \mathrm{~N} 1$; MOI, 0.5), or B/Bris (Victoria-lineage; MOI, 1). Viral gene expression was measured $8 \mathrm{~h}$ post infection and normalized to NT controls. e, A549 cells stably expressing hnRNP UL1 (top) or MECR (bottom) were infected with WSN PB2-FLAG (MOI 3, $8 \mathrm{~h}$ ). Protein localization was detected by immunofluorescence and nuclei were visualized with DAPI. Arrow indicates minor PB2 population consistent with previously reported patterns of mitochondrial localization. Scale bar indicates $20 \mu \mathrm{m}$. Data in a are mean \pm SEM of $n=3$ biological replicates. Comparisons were performed with two-way ANOVA with post hoc Fisher's LSD test. For $\mathbf{c}$ and d, data are mean \pm $\mathrm{SD}$ of $n=3$. Comparisons were performed with a two-tailed Student's t test (c) or a two-way ANOVA with post hoc Dunnett's multiple comparisons test $(\mathbf{e}) ;{ }^{*}, P<0.05$; ${ }^{\star *}$, $P<0.01$; ${ }^{* \star *}, P<0.001 ;{ }^{* * * *}, P<0.0001$; ns, not significant.

hnRNP UL1 reduced viral gene expression during infection for all strains, as did our control target 
NXF1. Knockdown of MECR again increased infection by WSN, but not CA04. B/Bris was even more impacted by MECR knockdown with a $\sim 4$-fold increase in viral gene expression compared to 1.5 to 2-fold effect seen for WSN. We also measured viral gene expression during a single round of infection. Similar to results with viral replication, viral gene expression was reduced when hnRNP UL1 was knocked down and increased when MECR was knocked down, and this was independent of the identity of PB2 residue 627 (Supp. Fig. 2c,d). These data suggest that hnRNP UL1 functions as a pro-viral factor, contrasting with the anti-viral activity of MECR. Further, hnRNP UL1 was important for all influenza $A$ and $B$ virus strains we tested, whereas the inhibitory activity of MECR showed strain-specific effects.

hnRNP UL1 interacts with the viral replication machinery to promote replication. hnRNP UL1 is an RNA-binding protein that plays a role in nucleocytoplasmic RNA transport as well as DNA end resection signaling during double strand break repair (Gabler et al., 1998; Polo et al., 2012). It was first characterized through its function with another virus, binding to the early protein E1B-55 kD from adenovirus. As a member of the hnRNP family of proteins that are well-characterized regulators of pre-mRNA processing, hnRNP UL1 has also been shown to interact with NXF1 and NS1-BP, proteins that help coordinate export of influenza viral mRNAs and splicing of the viral genome, respectively (Bachi et al., 2000; Satterly et al., 2007; Tsai et al., 2013). Consistent with its known function, immunofluorescence assays showed that
hnRNP UL1 is present primarily in the nucleus of infected cells where it co-localized with PB2 (Fig. 2e). Infection does not appear to change hnRNP UL1 localization. We tested interactions between the viral polymerase and endogenous hnRNP UL1. Cells were infected with WT virus or virus encoding PB2-FLAG and subject to FLAG immunoprecipitation. Endogenous hnRNP UL1 coprecipitated in the presence of PB2-FLAG, as did the polymerase subunit PB1, but none were presented in the control precipitation with untagged PB2 (Fig. 3a), demonstrating specific association with PB2 and confirming our ICC-MS.

Nucleocytoplasmic transport of viral mRNA is a major bottleneck for viral gene expression. There is a limiting amount of host NXF1 which chaperones mature viral mRNA to the nuclear basket for transport (Satterly et al., 2007). To test the importance of hnRNP UL1 and whether it is limiting, we stably over-expressed hnRNP UL1. Viral titers increased almost 3-fold in cells expressing more hnRNP UL1 (Fig. 3b). Similar results were demonstrated upon over-expression of NXF1 or TPR, which connects viral mRNA to the nuclear pore complex (Supp. Fig. 3b; (Delaleau and Borden, 2015)). The viral polymerase is an RNA-binding protein that exists both in a free form or incorporated into viral RNP with genomic RNA and NP. We performed a series of interactions studies to dissect the complexes that interact with hnRNP UL1 and the role of RNA in these interactions. PB2 interacted with hnRNP UL1 when co-expressed in 293T cells (Fig. 3c). When viral polymerase components PB1 and PA were additionally expressed in cells with hnRNP UL1,
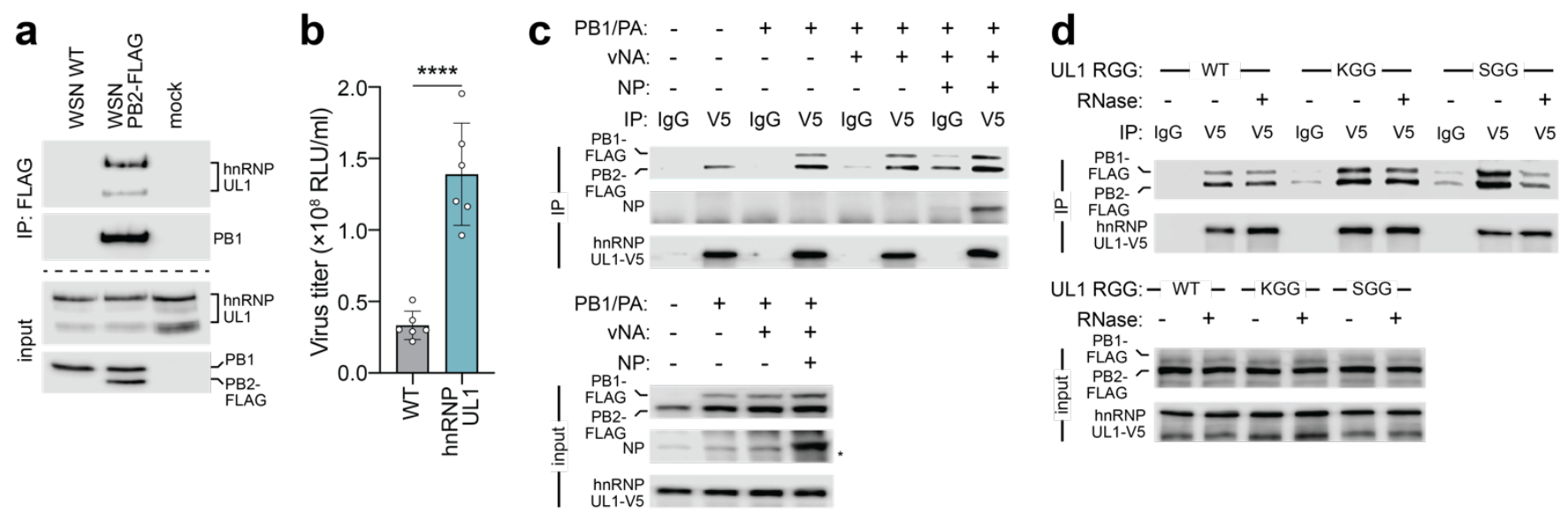

Fig. 3: Proviral hnRNP UL1 associates with influenza polymerase. a, Endogenous hnRNP UL1 co-precipitates with PB2 during infection. A549 cells were infected (MOI, $1 ; 18$ h) or mock treated, lysed and immunoprecipited. Proteins were detected by western blot. b, Viral titers were measured from wildtype or clonal A549 hnRNP UL1-V5 cells infected with WSN Nluc (MOI, $0.05 ; 24 \mathrm{~h}$ ). Mean \pm SD of $n=6$. Unpaired two-tailed t test; ${ }^{* * * *}, P<0.0001$. c-d, Association of viral polymerase with hnRNP UL1. c, 293T cells expressing PB2-FLAG and hnRNP UL1-V5 were immunoprecipitated with anti-V5 antibody or control IgG alone or co-expressing polymerase components PA and PB1-FLAG, viral RNA (vNA), and NP. Proteins were detected by western blotting. Asterisk indicates non-specific band. d, Co-immunoprecipitation assays were performed using WT hnRNP UL1 or mutants where the RNA binding motif "RGG box" was changed to disrupt PRMT-mediated methylation (KGG) or RNA interaction (SGG). RNase was included during immunoprecipitation where indicated. 
PB1 and PB2 were more effectively co-precipitated by hnRNP UL1, indicating that hnRNP UL1 interacts with the trimeric polymerase in the absence of other viral proteins or RNAs. Expressing genomic RNA in these cells did not increase the interaction between the polymerase and hnRNP UL1. However, including genomic RNA and NP, which permits formation of viral RNP, resulted in specific co-precipitation of NP. hnRNP UL1 is also an RNA-binding protein that forms multi-protein complexes. hnRNP UL1 binds RNA through its RGG box and methylation of arginine residues in the RGG box facilitates association with some of its protein partners (Gurunathan et al., 2015; Ozdilek et al., 2017). To evaluate these functions, we mutated hnRNP UL1 to eliminate RNA binding (RGG to $S G G$ ) or methylation of the RGG box (RGG to KGG) (Fig. 3d). Interactions between the viral polymerase and hnRNP UL1 were indistinguishable between WT, an RNAbinding mutant, or a methylation mutant. These mutations did not change the localization of hnRNP UL1, which remained localized to the nucleus (Supp. Fig. 3c). Exogenous RNase was added during immunoprecipitation to disrupt RNAmediated interactions. RNase treatment resulted in minor changes, but did not dramatically affect complex formation (Fig. 3d). Together, these results show that hnRNP UL1 enhances replication by binding to the viral polymerase, potentially increasing access to the NXF1-mediated export pathway.

MECR antiviral activity is independent from its role in mtFAS. MECR is an essential enzyme for mitochondrial fatty acid synthesis. The acyl carrier protein (ACP) encoded by NDUFAB1 scaffolds each enzymatic step in the mitochondria with MECR performing the final step converting trans2-enoyl-ACP to acyl-ACP (Fig. 4a; reviewed in (Nowinski et al., 2018)). Acyl-ACP feeds into oxidative phosphorylation or is converted to octanoyl-ACP then lipoic acid for protein lipoylation or use in the TCA cycle. To test whether the mtFAS pathway itself has an antiviral role, we knocked down ACP, which potently limits lipoic acid accumulation (Feng et al., 2009). Contrary to the antiviral activity of MECR, loss of ACP did not cause a significant change in viral titers (Fig 4b). Combined knockdown of MECR and ACP maintained the increased replication associated with MECR knockdown but did not have any additive effects. We additionally targeted mtFAS by treating infected cells with $\mathrm{C} 75$, a drug that targets
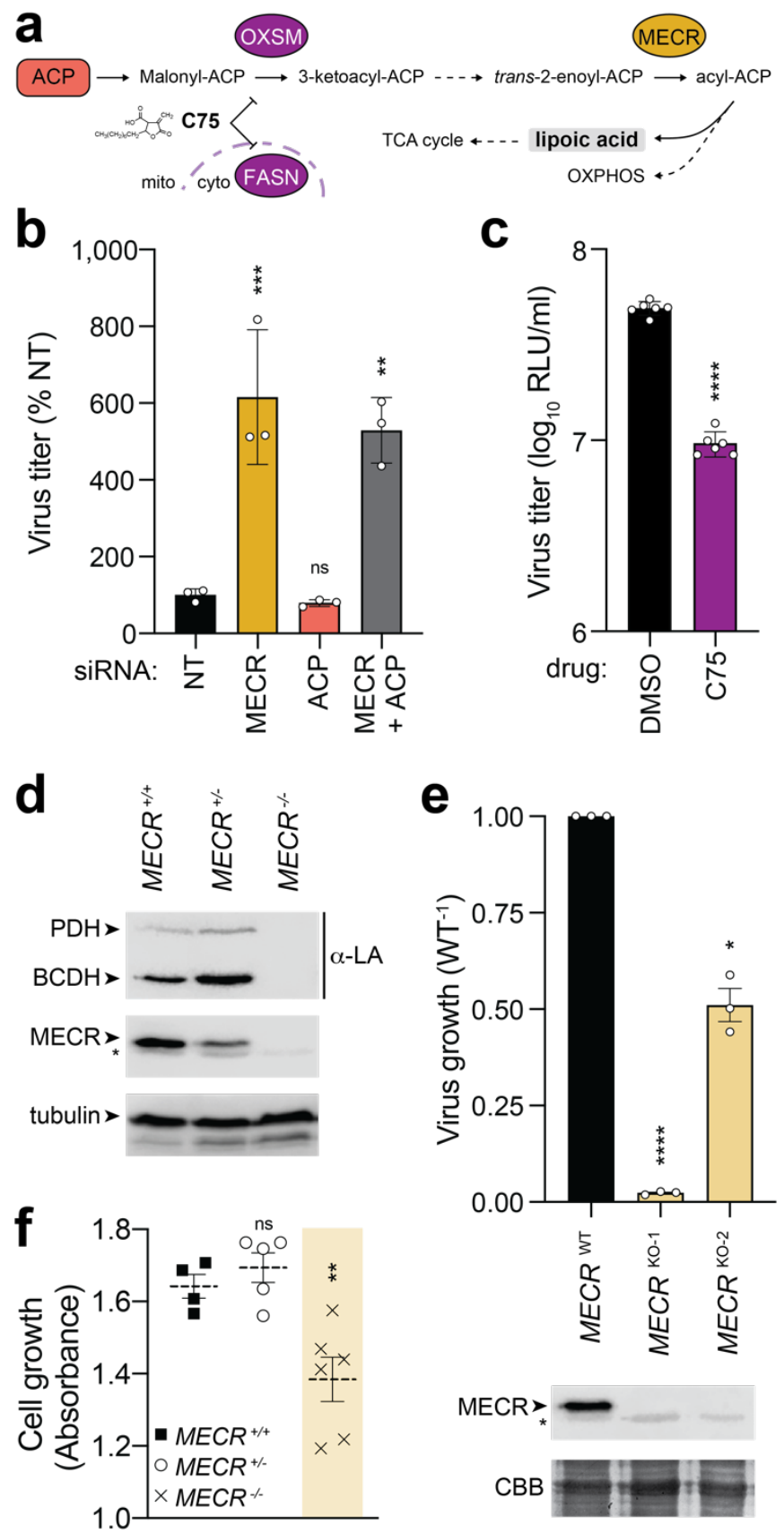

Fig. 4: Modulating the critical mtFAS enzyme MECR alters mtFAS output and virus growth. a, Focused snapshot of the mtFAS pathway. Acyl-carrier protein (ACP) and MECR were experimentally probed by knockdown or knockout, whereas 3-Oxoacyl-ACP Synthase, Mitochondrial (OXSM) and fatty acid synthase (FASN) were inhibited with the drug C75. b, A549 cells were treated with siRNA targeting MECR, ACP, both, or a non-targeting (NT) control prior to infection with WSN NLuc virus (MOI, $0.05 ; 24 \mathrm{~h}$ ). Viral titer in supernatants was determined and normalized to NT. Mean \pm SD of $n=3$. One-way ANOVA with post hoc Dunnett's multiple comparisons test; ${ }^{\star *}, P<0.01 ;{ }^{* * *}, P<0.001$; ns, not significant. c, Viral yield was measured from A549 cells treated with C75 or DMSO control prior to infection with WSN NLuc (MOI, $0.05 ; 24 \mathrm{~h}$ ). Mean \pm SD of $n=6$. Unpaired two-tailed t test; ${ }^{* * *}, P$ $<0.0001$. d, Production of lipoylated pyruvate (PDH) and branched chain dehydrogenases $(\mathrm{BCDH})$ was assessed in WT $\left({ }^{+/+}\right)$, heterozygous $\left({ }^{+/}\right)$, and homozygous $\left(^{-l-}\right)$ MECR knockout A549 cells by western blotting with anti-lipoic acid ( $\alpha$-LA). e, Virus replication was measured in A549 cells (MOI, 0.05; $24 \mathrm{~h}$ ). Replication in MECR knockout clones KO-1 and KO-2 was normalized to wildtype (WT) A549 cells. Mean \pm SEM of biological replicates $(n=3)$ normalized to WT. MECR expression was monitored by western blotting, whereas Coomassie brilliant blue (CBB) staining was used as a loading control. Asterisks indicate non-specific bands. $f$, Growth of clonal WT, heterozygous, or homozygous MECR knockout A549 cells was measured over three days. Mean \pm SEM of $n=4-6$ clones. One-way ANOVA with post hoc Dunnett's multiple comparisons test; ${ }^{*}, P<0.01$; ns, not significant. 
OXSM in the mtFAS pathway as well as FASN that directs cytoplasmic fatty acid synthesis (Chen et al., 2014). Whereas specific knockdown of MECR increased replication, complete loss of fatty acid synthesis reduced viral titers, likely by disrupting cellular metabolism (Fig. 4b-c).

To further dissect the antiviral role of MECR and any contribution from mtFAS, we generated MECR knockout A549 cells. MECR is not a strictly essential gene (Chen et al., 2017), but deletions in mice are embryonically lethal and it may be necessary for mammalian skeletal myoblasts (Nair et al., 2017; Nowinski et al., 2020). We recovered A549 cells with edited heterozygotic and homozygotic knockout alleles (Supp. Fig 4a). Loss of MECR completely disrupted mtFAS, as indicated by loss of lipoylated proteins (Fig. 4d). mtFAS remained intact in a heterozygotic cell clone. Our knockdown experiments predicted that loss of MECR would enhance virus production, yet we observed that two independent MECR knockout cells uniformly produced less virus than wildtype (Fig. 4e). We noted that MECR knockout cells grew slower compared to WT or MECR heterozygotic cells (Fig. 4f). The limited virus growth in knockout cells is likely not connected to the antiviral activity of MECR, but is instead due to cellular defects in mtFAS that manifest as a loss of mitochondrial lipoic acid synthesis, slower cell growth, and defects in respiratory chain complex integrity (Fig. 4e,f; (Feng et al., 2009)). These data suggest that MECR antiviral activity is independent from its normal role in supporting cellular metabolism.

A cryptic isoform of MECR escapes the mitochondria to bind the polymerase and exert antiviral activity. Our ICC-MS results provided high confidence identification of MECR as a PB2 interactor (Supp. Table 1). While a minor population of PB2 localizes to the mitochondria (Fig. 2d; (Carr et al., 2006)), we did not detect robust interactions between the polymerase and endogenous MECR (data not shown). Analysis of RNA-seq data from influenza virus infected A549 cells revealed that $29 \%$ of MECR transcripts $( \pm 5 \%$, $n=3$ ) have an alternative splicing pattern. The alternative splice form utilizes an upstream splice acceptor site between exon 1 and 2 (Fig. 5a). The splicing pattern introduces a stop codon in-frame with the original MECR start codon to create an upstream open reading frame that promotes initiation at the downstream start site M77 in MECR. Translation from the downstream start codon skips coding sequences for the mitochondrial targeting signal in MECR to produce cytoplasmic MECR (cMECR; (Kim et al., 2014)). Full-length MECR displayed a mitochondrial-like subcellular organization, whereas expressing CMECR resulted in diffuse staining throughout the cytoplasm and nucleus, consistent with the absence of a mitochondrial targeting sequence (Fig. 5b). Comparing infected and uninfected cells indicated that infection did not impact MECR distribution.

The ICC-MS results could not distinguish whether PB2 interacted with MECR or CMECR. We tested interactions between the viral polymerase and either MECR or CMECR by coimmunoprecipitation (Fig. 5c). MECR was strongly expressed, but precipitated very small amounts of the co-expressed viral PB2, and no PB2 when the polymerase or RNP components were additionally expressed. cMECR, by contrast, was poorly expressed but robustly interacted with PB2 in the absence or presence of viral polymerase and RNP components. To evaluate if infection played a role in this interaction parallel immunoprecipitations were performed in cells co-expressing RNP and MECR or CMECR that were additionally infected with influenza virus (Fig. 5d). Infection did not change the interactions. The minor co-purification of polymerase with MECR is likely due to leaky expression from M77 in MECR, recreating cMECR from the MECR transcript. Given that CMECR interacted with the viral polymerase, we asked whether it conferred the antiviral phenotype revealed by our knockdown experiments (Fig. 2a, c). Viral titers were measured following infection of clonal A549 cells expressing MECR or cMECR. Expression of MECR did not alter replication, whereas minimal expression of cMECR resulted in a significant reduction in viral titers (Fig. 5e). Collectively, these data suggest that cMECR, expressed from an alternatively spliced isoform, interacts with the viral polymerase to exert antiviral activity. Given that cMECR cannot localize to the mitochondria, it likely does not have a role in mtFAS, supporting our prior observations that the antiviral activity of MECR is independent of mtFAS.

\section{Repairing $M E C R^{-/}$cells with the yeast homolog assigns antiviral activity to CMECR MECR plays an essential role in mtFAS, making it challenging to cleanly delineate MECR function during mtFAS from the putative antiviral role of cMECR. Indeed, mtFAS deficiency in $\mathrm{MECR}^{-/-}$cells resulted in a generic defect in cellular metabolism}



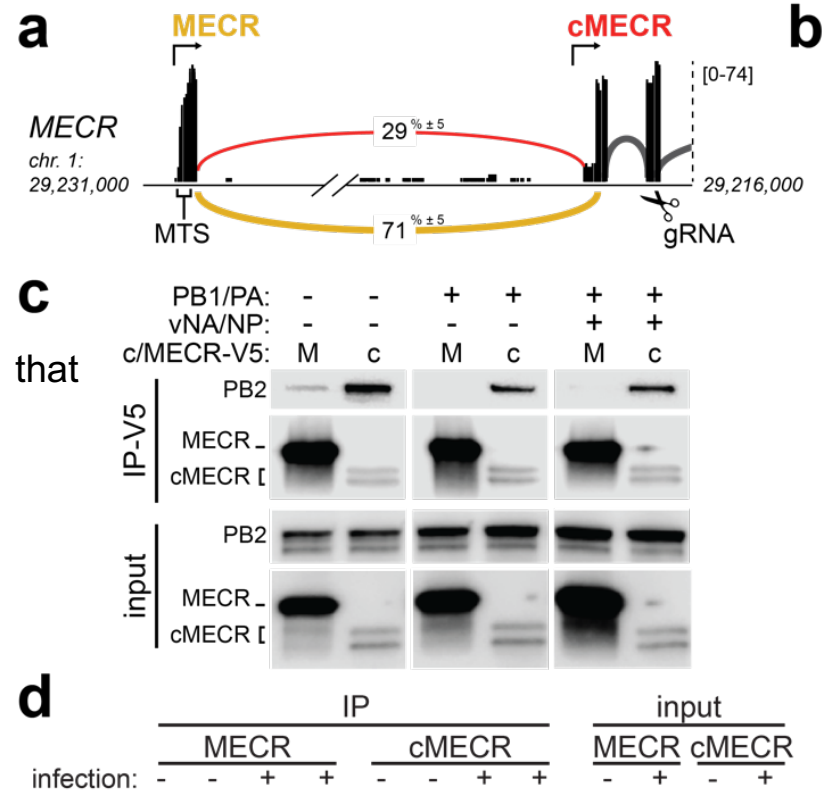

IP: IgG V5 IgG V5 $\lg G$ V5 $\lg G$ V5
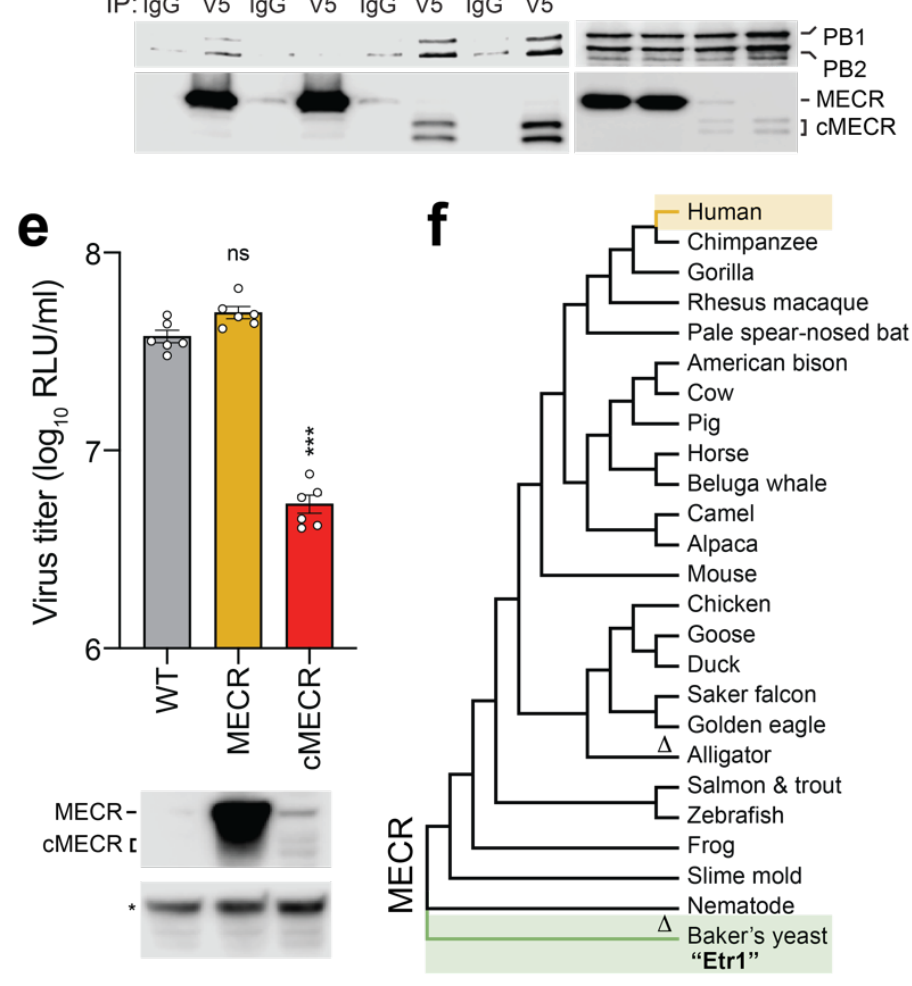

nonspecifically reduced cell growth, and consequently viral replication (Fig. 4). We therefore sought to repair the mtFAS pathway in $\mathrm{MECR}^{-/}$cells by expressing a homolog of MECR that lacks cMECR. cMECR translation relies on the start codon at M77, which is surprisingly wellconserved (Fig. 5f). Phylogenetic analysis from vertebrates suggests that CMECR was acquired after the divergence of animalia from fungi (Fig. 5f). Only the ancestral homolog of MECR from baker's yeast (Saccharomyces cerevisiae) and, interestingly, salmon (Salmo salar) and trout
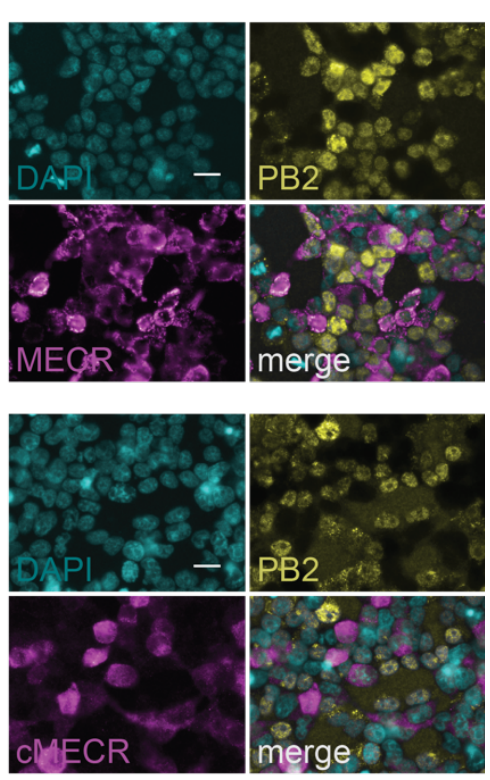

9 +yeast Etr1

MECR: WT KO-1 KO-2
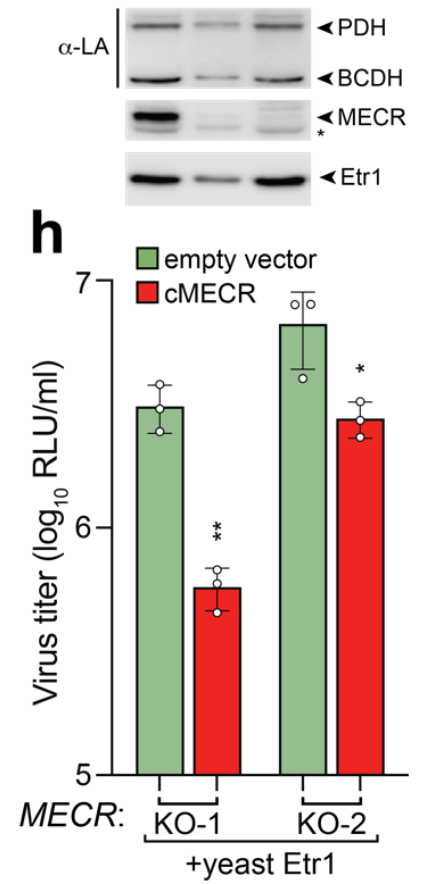

Fig. 5: The alternative splice variant CMECR exerts antiviral activity independent of mtFAS. a, Sashimi plot of RNA-seq data from A549 cells showing alternative 3 ' splice site utilized by transcripts that do not translate the mitochondrial targeting signal (MTS) in exon 1. Numbers embedded in yellow (MECR) and red (cMECR) curves indicate percentage of each splicing event as a total of all exon-joining reads. Arrows indicate transcriptional start sites and gRNA denotes region targeted in CRISPR-Cas9 editing. b, 293T cells expressing MECR or CMECR were infected with influenza PB2-FLAG virus (MOI, 3; $8 \mathrm{~h}$ ). Subcellular localization of PB2, MECR or CMECR were determined by immunofluorescence microscopy. Scale bar indicates $20 \mu \mathrm{m}$. c-d, Viral polymerase associates with CMECR during infection. c, PB2-FLAG and V5tagged MECR or CMECR were expressed in 293T cells with or without the other polymerase (PB1/PA) or VRNP (vNA/NP) components. d, PA, PB1FLAG, PB2-FLAG and MECR or CMECR were expressed in $293 \mathrm{~T}$ cells. Where indicated, cells were also infected with WSN (MOI, 10; $6 \mathrm{~h}$ ). Cells were lysed and subject to immunoprecipitation with anti-V5 antibody or IgG controls. Proteins were detected by western blot. e, Replication of WSN NLuc was measured in WT or clonal A549 cells expressing MECR or CMECR. Protein expression from infected cells was analyzed by western blotting. Asterisk indicates a non-specific band used as a loading control. Mean \pm SD of $n=6$. One-way ANOVA with post hoc Dunnett's multiple comparisons test; $\star \star \star *$, $P<0.001$; ns, not significant. f, Phylogenetic maximum-likelihood analysis of MECR amino acid sequences. $\Delta, \quad$ sequences lacking conserved cMECR start codon (Met77, human numbering). g-h, The yeast MECR homolog Etr1 repairs mtFAS in MECR knockout cells. g, Lipoylation of pyruvate $(\mathrm{PDH})$ and branched chain dehydrogenases (BCDH) in WT or clonal MECR KO-1 and KO-2 cells complemented with $S$. cerevisiae Etr1 was assessed by western blotting whole cell lysate with anti-lipoic acid ( $\alpha-L A)$. $\mathbf{h}$, Etr1-expressing MECR knockout cells were transduced with lentivirus expressing CMECR or an empty vector control. Cells were subsequently infected with WSN NLuc (MOI, 0.05; 24 h) and viral titers were measured in supernatants. Mean \pm SD of $n=3$. Unpaired two-tailed t test; * $P<0.05$; ${ }^{* *}$, $P<0.01$.

(Salmo trutta), do not encode M77 and presumably do not produce cMECR.

To assess whether the antiviral activity of cMECR is independent from mtFAS and MECR in general, we complemented $M E C R^{-/}$cells by stably expressing the yeast homolog Etr1. Etr1 functioned in human cells to restore lipoic acid synthesis as indicated by the detection of lipoylated proteins (Fig. 5g). With mtFAS restored by Etr1, we could independently test the antiviral activity of cMECR. Ectopic cMECR expression markedly reduced viral replication in two independent 
knockout cell lines complemented with Etr1 (Fig. 5h). The genetic complementation approach also demonstrated that the anti-influenza activity of cMECR does not require the presence of full-length MECR, which is important considering MECR is known to homodimerize (Chen et al., 2008). Collectively, our results decouple the newly described function of CMECR from the canonical role of MECR, and suggest that an antiviral activity has been embedded within an important metabolic enzyme.

\section{Discussion}

Zoonotic influenza viruses must gain a foothold to replicate and then adapt to their new human hosts. Here we used ICC-MS to identify human proteins that specifically interact with avian polymerases containing avian or human-signature PB2 during infection. Network simulations and siRNA screening highlighted two key interactors, hnRNP UL1 and MECR. We showed that hnRNP UL1 supports viral replication and likely acts as part of a pathway commonly exploited by viruses to aid mRNA splicing and transport. MECR, by contrast, exerted antiviral activity suppressing viral gene expression and replication. MECR has a welldefined role in mitochondrial fatty acid synthesis, yet this pathway was not identified in our network analyses and mechanistic studies showed that the antiviral activity was independent of mtFAS. MECR and mtFAS were generically important for viral replication, in as much as they were necessary for proper mitochondrial metabolism and cell health. Instead, we assigned the antiviral activity to CMECR, an alternative splice variant that produces a cytoplasmic form of the protein lacking the mitochondrial targeting sequence. Using the yeast homolog Etr1 to supply the metabolic functions of MECR, we confirmed that antiviral activity is independent of mtFAS and lies solely within cMECR. Thus, a cryptic antiviral activity has been embedded within a key metabolic enzyme, possibly protecting it from viral countermeasures.

hnRNP family members, of which there are 37 in humans, broadly regulate host nucleic acid processes including chromatin organization, DNA damage repair, pre-mRNA processing and splicing, and mRNA nuclear export and subcellular transport (Busch and Hertel, 2012; Geuens et al., 2016). As a consequence, hnRNPs are frequent co-factors for viral replication, including hnRNP UL1 we characterized here (Meyer, 2016). hnRNP
UL1 contains RGG domains that can be methylated and are involved in RNA binding. However, neither of these activities was required for interaction with the viral polymerase (Fig. 3e). hnRNP UL1 also binds to NXF1, the major cellular factor involved in nuclear export of viral mRNAs (Bachi et al., 2000; Read and Digard, 2010; Satterly et al., 2007). We speculate that hnRNP UL1 enhances viral replication by bridging interactions between transcribing viral polymerases and NXF1 to facilitate mRNA export.

Mitochondria are a major subcellular location where host-pathogen conflicts play out: cellular IFN pathways use the mitochondrial membrane to assemble signaling hubs containing MAVS; mitochondrial nucleic acids can be unshielded to trigger the type-I IFN response, and miRNAs that can regulate IFN stimulated genes are embedded in mitochondrial genes (Dhir et al., 2018; Hou et al., 2011; Refolo et al., 2020; Sorouri et al., 2020). Knockdown of MECR in A549 MAVS, RIG-I, or PKR knockout cells still alleviates its antiviral activity (Supp. Fig. 4c), suggesting that the antiviral activity of MECR functions independent of innate sensing pathways that converge at the mitochondria. Instead, our data uncover another conserved mechanism where the metabolic enzyme MECR moonlights as the antiviral protein cMECR. CMECR is translated from an alternatively spliced transcripts that utilizes a downstream start codon to produce a protein lacking the mitochondrial targeting sequence. cMECR limits influenza $A$ and $B$ viruses, but not pandemic 2009 H1N1 (CA04) (Fig. 2e). WSN and $\mathrm{B} / \mathrm{Bris}$ are human origin strains, whereas the PB2 gene from CA04 is avian-like (Dawood et al., 2009). Human-derived PB2 genes localize to the mitochondrial matrix due to a mitochondrial targeting sequence in the $\mathrm{N}$-terminus; the targeting sequences are disrupted in avian PB2 proteins by an N9D polymorphism (Carr et al., 2006; Graef et al., 2010; Long and Fodor, 2016). Whether this contributes to differences in sensitivity to cMECR is unclear.

Phylogenetic analysis revealed that both MECR and cMECR are highly conserved in typical influenza hosts, including humans, birds, pigs, horses, and dogs, which made the loss of M77 and potentially cMECR in Salmo species interesting (Fig. 5f; amino acid numbering based on human MECR). Salmo species are hosts for infectious salmon anemia virus, an orthomyxovirus closely related to influenza virus. While they do not appear to utilize the alternative splice acceptor site that 
could create cMECR, they may still express a cMECR-like protein by initiating translation at the conserved M89 of MECR. But, this would be a distinct mechanism, such as leaky scanning, than the alternative splicing used for cMECR production in other vertebrates. Salmo do vary splicing between exon 1 and 2 by utilizing an alternative splice donor to create a splice isoform that encodes 26 amino acids not found in humans. It will be important to determine if Salmo MECR encodes a protein with antiviral activity, providing information on the antiviral function of MECR in different species and illuminating how conditions that alter splicing patterns or translation initiation sites stimulate or repress cMECR production.

The antiviral activity of CMECR is embedded within the essential metabolic functions of MECR, presenting a Corneillian dilemma for influenza virus. Targeting cMECR to alleviate its antiviral activity would disable MECR and mtFAS, a metabolic process important for cell viability and influenza virus output (Fig. 4). Conversely, leaving MECR and mtFAS intact would ensure cell health, but the virus then remains vulnerable to CMECR (Fig. 5). The host strategy of encoding an alternatively spliced antiviral protein that shares structure with an energetically important protein engineers a failsafe for the host, forcing the virus to make the difficult decision of antiviral antagonism or maximum biosynthetic power. It is likely that similar strategies litter host genomes to fortify antagonism from diverse pathogens.

\section{Methods}

Viruses, cells, plasmids, antibodies. Influenza viruses and plasmids were derived from A/WSN/33 (H1N1; WSN), A/green-winged teal/Ohio/175/1986 (H2N1; S009), A/California/04/2009 (H1N1; CA04), and B/Brisbane/60/2008 (B-Victoria lineage; B/Bris) (Karlsson et al., 2015; Larson et al., 2019; Mehle and Doudna, 2008, 2009). Recombinant virus was rescued by transfecting co-cultures of 293T and MDCK cells with pTM $\triangle$ RNP encoding WSN vRNA segments $\mathrm{HA}, \mathrm{NA}, \mathrm{M}$, and NS and the bi-directional pBD plasmids encoding vRNA and mRNA for PB1, PA, NP, and the indicated PB2 mutants (Mehle and Doudna, 2009; Neumann et al., 2005). WSN PB2-FLAG and WSN-PB2-627E-FLAG virus were previously described (Baker et al., 2018; Kirui et al., 2014). S009 PB2-FLAG and S009 PB2-627K-FLAG viruses contain NP and polymerase genes from S009 and the remaining segments from WSN and were constructed as previously described (Mehle and Doudna, 2009; Dos Santos Afonso et al., 2005). Nanoluciferase-expressing (NLuc) viruses were engineered to co-linearly express $P A$, a $2 A$ cleavage site, and NLuc (PASTN, referred to as WSN NLuc) on the third viral segment (Tran et al., 2013). CA04 NLuc and B/Bris NLuc were generated similarly (Karlsson et al., 2015; Larson et al., 2019). Viral stocks were amplified on MDBK cells and titered by plaque assay on MDCK cells. Influenza virus infections were performed by inoculating cells with stocks diluted in virus growth media (VGM; DMEM supplemented with penicillin/streptomycin, $25 \mathrm{mM} \mathrm{HEPES,}$ $0.3 \% \mathrm{BSA}$, and $0.25-0.5 \mu \mathrm{g} / \mathrm{ml}$ TPCK-trypsin).

VSV-G pseudotyped lentivirus was prepared by transfecting 293T cells with pMD2.G (Addgene 12259), pLX304 (Addgene 25890) encoding HNRNPUL-1-V5 or MECR-V5; HNRNPUL1-V5 and MECR-V5) and psPAX2 (Addgene 12260) or pMD2.G, pQCXIP encoding Etr1-HA (Clontech) and pCIG-B (Bock et al., 2000). Resultant viruses were used to transduce A549 cells. Cells were selected with blasticidin or puromycin to obtain stable expressing lines. Mammalian cells were grown in DMEM

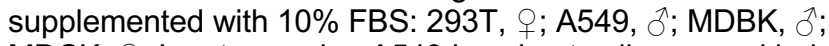
MDCK, \%. Innate sensing A549 knockout cells were a kind gift from $\mathrm{C}$. McCormick and described previously (Rahim et al., 2020). All cells were maintained at $37^{\circ} \mathrm{C}, 5 \% \mathrm{CO}_{2}$. Cell stocks were routinely tested for mycoplasma (MycoAlert, Lonza). Human cell lines were authenticated by STR analysis (University of Arizona Genetics Core).

A549 MECR knockout cells were generated using CRISPR-Cas9 with a single guide RNA targeting exon 3 of MECR (GTTGCACAGGTGGTAGCGGTGGG designed by crispr.mit.edu). Annealed tracrRNA/gRNA was complexed with Cas9 (Alt-R; IDT) and RNPs were electroporated (Lonza) into cells following manufacturer's instructions. Two days post nucleofection, bulk population (42\% editing efficiency by sanger sequencing and ICE analysis (Hsiau et al., 2018)) was single cell sorted into 96 well plates by FACS. Clones were screened by targeted next generation sequencing of genomic DNA (Genome Engineering \& iPSC Center, Washington University in St. Louis). Biallelic knockout cells were verified by western blot.

Cell proliferation assays were conducted over three days using CellTiter $96 \mathrm{AQ}$ reagent (Promega) at 24 or $72 \mathrm{~h}$ after seeding, incubating $2 \mathrm{~h}$, and measuring absorbance. Background absorbance at $630 \mathrm{~nm}$ was subtracted from 490 $\mathrm{nm}$. Measurements were performed in technical triplicate and activity from $72 \mathrm{~h}$ subtracted from $24 \mathrm{~h}$ to determine growth. pENTR HNRNPUL1 was generated using Gibson assembly from pDONR HNRNPUL1 obtained from DNASU.org (HsCD00719283). RNA binding mutants of HNRNPUL 1 were generated by synthesizing mutant RGG boxes (nucleotides encoding amino acids 612-658; IDT) where all arginines were replaced with serine (SGG) or lysine (KGG) and introduced using Gibson assembly (Gurunathan et al., 2015; Ozdilek et al., 2017). pDONR MECR was acquired from DNASU (HsCD00399762). pDONR cMECR was generated using iPCR to delete nucleotides encoding the first 76 amino acids of MECR. HNRNPUL1 and MECR were recombined into V5-tagged mammalian expression constructs by Gateway cloning into pcDNA6.2 (Invitrogen) and pLX304 (Addgene 25890). pQCXIP (Qiagen) Etr1-HA was generated by Gibson assembly using pDONR Etr1 (DNASU; ScCD00009122). pcDNA3 FLAG-TPR was acquired from Addgene (60882). pcDNA6.2 NXF1 was generated by Gateway cloning pENTR NXF1 (DNASU; HsCD00514182).

Antibodies used for blotting include monoclonal antiFLAG clones $1 / 27$ and 1/54 made in-house at Roche, antiFLAG M2-HRP (Sigma A8592), polyclonal anti-MECR (Proteintech 14932-1-AP or Atlas Antibodies HPA028740), polyclonal anti-hnRNP UL1 (Proteintech 10578-1-AP), polyclonal anti-V5 (Bethyl Labs A190-120A), monoclonal anti-V5-HRP (clone V5-10, Sigma V2260), polyclonal anti- 
PB1 (Mehle and Doudna, 2009); monoclonal anti-tubulin (clone DM1A, Sigma T6199); polyclonal anti-lipoic acid (Calbiochem 437695); monoclonal anti-HA-HRP (clone 3F10, Sigma 12013819001), polyclonal anti-rabbit-HRP (Sigma A0545); and rabbit IgG (2729, Cell Signaling Technology). Antibodies used for immunofluorescence include mouse monoclonal anti-FLAG (Sigma F1804), rabbit polyclonal antiV5 (Bethyl Labs A190-120A), goat anti-mouse Alexa Fluor 594 (Invitrogen A-11032), goat anti-rabbit Alexa Fluor 488 (Invitrogen A-11008).

Immuno-competitive capture (ICC). ICC was performed as previously published (Meistermann et al., 2014) with the following modifications. Anti-FLAG clone $1 / 27$ was coupled to Affi-Gel 10 resin following the manufacturer's instructions (Bio-Rad). A549 cells were inoculated at an $\mathrm{MOI}$ of 0.2 with S009 PB2-FLAG or S009 PB2-627K-FLAG in a $10 \mathrm{~cm}$ dish, 5 dishes per replicate, 3 biological replicates. Infections were allowed to proceed for $24 \mathrm{~h}$. Cells were combined and lysed in $1 \mathrm{ml}$ co-IP buffer (50 mM Tris, pH 7.4, $150 \mathrm{mM} \mathrm{NaCl}, 0.5 \%$ NP-40, 1X cOmplete protease inhibitor (Roche)) and divided into equivalent fractions for ICC. Lysates were incubated by rocking for $3 \mathrm{~h}$ at $4{ }^{\circ} \mathrm{C}$ with free competing antibody (antiFLAG 1/27) where applicable, then immunoprecipitated for $16 \mathrm{~h}$ with $1 / 27$ antibody coupled to Affi-Gel 10 resin. After immunoprecipitation, samples were washed four times with co-IP buffer and eluted in Laemmli buffer at $70^{\circ} \mathrm{C}$ for $10 \mathrm{~min}$. Samples were then transferred to new tubes and boiled for 10 min with $0.1 \mathrm{M} \mathrm{DTT} .10 \%$ of the immunoprecipitates were separated by SDS-PAGE and analyzed by western blotting using 1/54 anti-FLAG antibody or silver stained.

Mass spectrometry (MS). The remaining $90 \%$ of the ICC sample was separated on a $4-20 \%$ Tris-Glycine SDS-PAGE gel and stained with Coomassie blue. Lanes were cut from the gel and processed for in-gel digestion. Samples were analyzed with a nanoflow Easy-nLC 1000 system (Proxeon) connected to an Orbitrap Fusion Tribrid mass spectrometer and equipped with an Easy-spray source (Thermo Fisher Scientific). Samples were re-suspended in LC-MS buffer (5\% formic acid/ $2 \%$ acetonitrile), concentrated on an Acclaim PepMap C18 trapping column $(75 \mu \mathrm{m} \times 20 \mathrm{~mm}, 5 \mu \mathrm{m}$ particle size), and peptides separated on an Acclaim PepMap C18 EASY-spray column $(75 \mu \mathrm{m} \times 500 \mathrm{~mm}, 2 \mu \mathrm{m}$ particle size $)$ heated at $45^{\circ} \mathrm{C}$ using the following gradient at $300 \mathrm{~nL} / \mathrm{min}: 7-$ $50 \%$ B in $45 \mathrm{~min}, 50-80 \%$ B in $2 \mathrm{~min}, 80 \% \mathrm{~B}$ for $13 \mathrm{~min}$ (buffer A: $0.1 \%$ formic acid; buffer B: $0.1 \%$ formic acid/acetonitrile). The instrument was set to collect Orbitrap MS1 scans over a mass range from $\mathrm{m} / \mathrm{z} 300$ to 1500 using quadrupole isolation, a resolution of 120000 (at $\mathrm{m} / \mathrm{z} 200)$, an automatic gain control (AGC) target value of $2 \times 10^{5}$, and a maximum injection time (IT) of $100 \mathrm{~ms}$. Using datadependent acquisition (DDA) with a cycle time of $3 \mathrm{~s}$ between two MS1 scans, the most intense precursor ions with a minimum intensity of $5 \times 10^{3}$, were mono-isotopically selected for high-energy collision dissociation (HCD) using a quadrupole isolation window of $0.7 \mathrm{Th}, \mathrm{AGC}$ target of $1 \times 10^{4}$, maximum IT of $35 \mathrm{~ms}$, collision energy of $30 \%$, and ion trap readout with rapid scan rate. Charge states between 2 and 6 and only one per precursor was selected for MS2. Already interrogated precursor ions were dynamically excluded for 20 $\mathrm{s}$ using $\mathrm{a} \pm 10 \mathrm{ppm}$ mass tolerance.

MS raw files were processed for label free quantification using Progenesis QI 2.1 (Nonlinear Dynamics) and ions $\mathrm{m} / \mathrm{z}$ values were aligned to compensate for drifts in retention time between runs (maximum charge state set at +5 ). Peptides and proteins were identified by searching data with Mascot Server 2.5.1 (Matrix Science) together with the UniProt human (May 2016 release, 20,201 sequences) and the influenza S009 viral (12 sequences) protein databases. Searches used trypsin/ $P$ as an enzyme, a maximum of two missed cleavage sites, and $10 \mathrm{ppm}$ and $0.5 \mathrm{Da}$ as the precursor and fragment ion tolerances, respectively. Carbamidomethylated cysteines $(+57.02146 \mathrm{Da})$ were set as static while oxidized methionines $(+15.99492 \mathrm{Da})$ were set as dynamic modifications. The specFDR was restricted to $1 \%$ by performing a target-decoy search using a concatenated decoy database. Peptide extracted ion chromatograms (EICs) were used to determine peptide amounts. Data normalization was performed in Progenesis by applying a scalar multiple to each feature abundance measurement with the assumption that most peptide ions do not change in abundance (similar abundance distributions globally.

Data were quality controlled by assessing sample distribution and performing a principal component analysis. One outlier sample (Dose 0) was removed from the PB2627K experiment, based on Mahalanobis distance of the first 3 principal components (Varmuza and Filzmoser, 2016). Specific interacting proteins should decrease in relative abundance with increased concentration of the free competitor antibody; these displaced proteins are determined as previously described (Augustin et al., 2013). Briefly, a linear model is fit on the $\log _{2}$-transformed relative abundance values for each protein with the free competitor compound concentration. Then, monotonic contrasts are used to compare the protein abundance values above and below each concentration point (Bretz and Hothorn, 2001; Stewart and Ruberg, 2000). The maximum t-statistic from each series is determined with a moderated t-test (Smyth, 2004). Model fitting and post-hoc contrast tests are performed with limma (Ritchie et al., 2015). Significance of the displacement is assessed using permutation tests, where concentration labels were permuted 1000 times based on the step-down minP algorithm (Westfall and Young, 1993) modified for onesided tests, and adjusted for multiple testing (Benjamini and Hochberg, 1995). Proteins with adjusted $p$-values below $5 \%$ are considered specific binders. Computations were performed in R (R Core Team, 2019).

Network Analysis. Network analysis was formulated as a minimum-cost flow optimization problem inspired by ResponseNet but customized for our application (YegerLotem et al., 2009). Human protein-protein interactions were obtained from the STRING database (version 10.5), using only interactions supported by experimental evidence (Szklarczyk et al., 2015). PB2 interactors identified by ICCMS were designated as source nodes and the influenza host factors as target nodes in the STRING network. The host factors came from our prior genetic screens (Larson et al., 2019; Tran et al., 2020) and published RNA interference screens (Brass et al., 2009; Hao et al., 2008; Karlas et al., 2010; König et al., 2010; Shapira et al., 2009). All human gene symbols were mapped to Ensembl protein identifiers. Proteins not present in this version of the STRING interaction network (i.e. PB2 interactors ABLIM1, EPDR1, and some influenza host factors) and TMEM106B were not included in the network analysis, leaving 23 source nodes and 2,179 target nodes.

The goal in the minimum-cost flow problem is to transport units of flow from a source node $S$ in a network to a target node $T$ (Yeger-Lotem et al., 2009). $S$ and $T$ are special 
nodes that are added to the network and do not represent proteins. $S$ has outgoing edges to all of the protein source nodes, the PB2 interactors. $T$ has incoming edges from all the protein target nodes, the host factors. Flow can move from node to node through edges in the network. The total amount of flow to transport from $S$ to $T$ is fixed. However, each edge has its own cost associated with transporting a unit of flow over that edge and a capacity that limits how much flow it can transport. Flow was assigned to edges such that the total cost of transporting the fixed amount of flow is minimized. Additional constraints require that the total flow into a protein node in the network (that is, all nodes except $S$ and $T$ ) equals the total flow out of that node, the total flow from $S$ equals the total flow into $T$, and the flow assigned to each edge is non-negative and less than or equal to the edge's capacity. Solving the minimum-cost flow problem assigns how much flow each edge transports. The edges with positive flow compose a subnetwork, and in our application that subnetwork may comprise a predicted host influenza response pathway.

The standard minimum-cost flow problem was adjusted here to ensure that not all flow can be transported through one source or one target and a minimum number of sources and minimum number of targets that must transport positive flow was specified. The total flow to transport is then the product of these minimums. The network edges from $S$ to the sources had capacity equal to the minimum number of targets. The network edges from the targets to $T$ had capacity equal to the minimum number of sources. The protein-protein interaction edges had capacity equal to the total amount of flow, which did not constrain how much flow could be transported. The protein-protein interaction edges also had costs derived from STRING. The cost was one minus the STRING weight for the interaction, such that low confidence edges have higher costs. This construction guarantees that if a feasible solution exists, it will include at least as many sources and targets as requested.

Furthermore, the solution will use the most confident protein interaction edges to transport that flow. If multiple equallygood solutions exist, the solver selected one arbitrarily. If a solution could not be found, we reduced the minimum number of sources and targets. For the influenza network analysis, the minimum number of sources was set to 23 and the minimum number of targets to 200 . This number of targets produced subnetworks that had multiple PB2 interactors in each connected component, which we also refer to as modules, as opposed to subnetworks that placed each source in its own connected component. The SimpleMinCostFlow solver from the ortools Python package (version 6.10.6025,

https://developers.google.com/optimization) was used to solve the minimum-cost flow instances. Our Python code is available from https://github.com/gitter-lab/influenza-pb2.

Two control analyses were conducted to assess the significance of the protein interaction subnetwork from the minimum-cost flow solution. Both controls solve the minimum-cost flow problem many times using randomized input data that is not relevant to influenza $A$ virus. If a protein belongs to both the influenza virus subnetwork and these control subnetworks, it may have been selected due to properties of the STRING network rather than influenza relevance. We defined an empirical $P$ value for each node in the influenza subnetwork as the number of times that node appears in a control subnetwork divided by the number of control runs. We executed 1,000 control runs. The simulated control sampled 23 source nodes, the number of real PB2 interactors, uniformly at random from all nodes in the STRING network. It used the real influenza host factors as target nodes. The second type of control uses an alternative virus, hepatitis $C$ virus, as the input. The alternative control sampled 23 source nodes from the 1,864 human proteins that interact with the hepatitis $C$ virus nonstructural protein $5 A$ as sources (Meistermann et al., 2014). It used hepatitis C host factors from a CRISPR screen as targets (Marceau et al., 2016).

The node sizes in the influenza subnetwork visualizations were scaled to be proportional to the node's negative $\log _{10} P$ value from the simulated and alternative controls. If a node's empirical $P$ value was 0 , we set it to 0.001 for this visualization. In addition, subnetwork regions were annotated with enriched GO terms using the gprofilerofficial Python package (version 0.3.5) for gene set enrichment analysis on each connected component of the subnetwork (Raudvere et al., 2019). Each connected component-GO term enrichment result was ranked by a score that incorporated the negative $\log _{10} P$ value of the enrichment, the depth of the GO term in the biological process ontology, and the fraction of nodes in the connected component annotated with the GO term. The combined score emphasizes more specific GO terms that have statistically significant enrichment and cover a large fraction of nodes. For each connected component, we assigned the GO term with the largest combined score that had not already been assigned to a different connected component. The influenza subnetworks were visualized with Graphviz (Gansner and North, 2000).

siRNA screening and infection experiments. A549 or 293T cells were reverse transfected with $25 \mathrm{nM}$ siRNA (SMARTpool, Horizon) using siQuest or X2 (Mirus) in 96-well plates for $48 \mathrm{~h}$. Cells were inoculated with PASTN virus at an $\mathrm{MOI}$ of 0.1 (single cycle infection, $8 \mathrm{~h}$ ) or 0.05 (multicycle infection, $24 \mathrm{~h}$ ). Single cycle monolayers were seeded in white bottom plates and read directly for NLuc activity (Promega) on a Synergy HT plate reader (BioTek). Multicycle experiments were seeded in clear bottom plates and observed for siRNA toxicity. Supernatants were collected and titrated by infecting MDCK cells (white bottom 96-well plates) for $1 \mathrm{~h}$ with $20 \mu \mathrm{l}$ supernatant, washing twice with VGM, and incubating for $8 \mathrm{~h}$. Luciferase activity was read as above. Single cycle infections were also performed with CA04 (MOI, 0.5 ) and B/Bris (MOI, 1) PASTN viruses as above. Virus gene expression (single cycle infection) and titer (multicycle infection) were normalized to non-targeting siRNA control. For knockdown during wildtype virus infection, A549 cells were forward transfected for two days with $25 \mathrm{nM}$ siRNA in 24-well plates and infected with WSN (MOI, 0.01) for $24 \mathrm{~h}$. Supernatants were titrated by plaque assay on MDCK cells. For overexpression experiments, 293T cells were reverse transfected with hnRNP UL1, NXF1, or TPR using TransIT-2020 (Mirus) in 96-well plates for $24 \mathrm{~h}$. Transfected cells were infected with PASTN virus at an MOI of 0.01 for 24 $\mathrm{h}$, and supernatants titrated as above. A549 cells stably overexpressing hnRNP UL1, MECR or CMECR, or MECR knockout cells overexpressing Etr1-HA were clonally isolated and analyzed for V5 or HA-tagged protein expression, respectively. Etr1-complemented cells were then transduced with cMECR-containing lentiviruses and polyclonal blasticidin-resistant cells used for experimental analysis. A549 overexpression cells were infected with PASTN virus in 96 -well plates for $24 \mathrm{~h}$ at an MOI of 0.05 . Supernatants were titrated as above. 
Infection experiments analyzing the role of mtFAS were performed by treating A549 cells with siRNAs for $48 \mathrm{~h}$ in 96-well plates with $50 \mathrm{nM}$ non-targeting control siRNA, 25 nM non-targeting control mixed with 25 nM MECR siRNA, 25 nM non-targeting control mixed with 25 nM NDUFAB1 (ACP) siRNA, or $25 \mathrm{nM}$ of MECR and ACP siRNAs, followed by multicycle analysis with WSN PASTN as described above. For drug treatment, A549 cells were seeded in 96-well plates and treated with DMSO or $50 \mu \mathrm{M} \mathrm{C75}$ (Sigma C5940) for 24 $\mathrm{h}$, infected with WSN PASTN (MOI, 0.05; $24 \mathrm{~h}$ ) in the presence of DMSO or $50 \mu \mathrm{M} \mathrm{C75}$, and titrated as above.

\section{Co-immunoprecipitations. Interactions between} endogenous hnRNP UL1 and PB2 were tested by coimmunoprecipitation. A549 cells were infected with WSN PB2-FLAG (10 cm dish; MOI, 1; $18 \mathrm{~h}$ ), lysed in co-IP buffer, lysates immunoprecipitated overnight with anti-FLAG resin (M2, Sigma), and co-precipitating hnRNP UL1 was detected by western blot. For V5 immunoprecipitations, mammalian expression plasmids encoding PB2-FLAG, PB1-FLAG, PA, and HNRNPUL1-V5 or MECR-V5 were forward transfected into 293T cells using PEI (PEI MAX, Polysciences; 6-well plates). Cells were lysed in co-IP buffer, immunoprecipitated with anti-V5 antibody, and co-precipitating viral proteins were detected by western blot. To test the effect of viral RNA on polymerase:hnRNP UL1 interactions, plasmids expressing vNA or NP were included where indicated. Cells were lysed two days post-transfection in co-IP buffer with or without $1 \mu \mathrm{l}$ RNase A (Thermo Scientific). Interactions with MECR during infection were tested by forward transfecting 293T cells with mammalian expression plasmids encoding PB2-FLAG, PB1FLAG, PA, and MECR-V5 or CMECR-V5 for $42 \mathrm{~h}$ and infecting with WSN (MOI 10; $6 \mathrm{~h}$ ) followed by lysis. Lysates were incubated with 1.5 ug polyclonal anti-V5 or IgG for $1 \mathrm{~h}$ and captured with protein A agarose resin (P2545, Sigma) for $30 \mathrm{~min}$. Immunoprecipitates were recovered, washed four times with co-IP buffer and eluted by boiling in Laemmli sample buffer. Samples were separated by SDS-PAGE and analyzed by western blotting. Chemiluminescent images were captured on an Odyssey Fc Imager and quantified using Image Studio v5.2.5 (LI-COR). At least two biological replicate experiments were performed.

Immunofluorescence. 293T cells were forward transfected in 48-well plates with plasmids expressing V5-tagged hnRNP UL1, MECR or CMECR and $48 \mathrm{~h}$ later infected with WSN PB2-FLAG (MOI, 3; 8 h). A549 cells stably expressing hnRNP UL1 or MECR were seeded on coverslips in 12-well plates and infected with WSN PB2-FLAG (MOI, 0.5; $8 \mathrm{~h}$ ). Monolayers were fixed with $4 \%$ paraformaldehyde in PBS for $10 \mathrm{~min}$, quenched and permeabilized with $0.1 \mathrm{M}$ glycine + $0.1 \%$ Triton X-100 in PBS for 5 min, and blocked with 3\% BSA in PBS for 30 min at room temperature. Primary and secondary antibodies were sequentially incubated for $1 \mathrm{~h}$ each at room temperature at $1 \mu \mathrm{g} / \mathrm{ml}$ in blocking buffer. DAPI was added to 293T cells during secondary antibody incubation. Coverslips were mounted in medium containing DAPI (Vector Laboratories, $\mathrm{H}-1200)$. Images captured using 20X objectives on an EVOS FL Auto (ThermoFisher) and processed in Adobe Photoshop CC.

RNA-sequencing. RNA was isolated from A549 cells that were mock treated, interferon- $\beta$ treated $(250 \mathrm{U} / \mathrm{ml}$ for $8 \mathrm{~h})$ or infected with influenza WSN (MOI 0.02 for $24 \mathrm{~h}$ ) using TRIzol (Invitrogen). Biologic triplicate sample RNA was sent for library preparation and paired-end RNA-seq by Novogene
(SRA\# TBD). Sequences were trimmed with BBDuk in the BBMap suite and aligned to the human genome (hg38) with HISAT2 (Bushnell, 2015; Kim et al., 2019). Splicing events in the MECR locus were visualized in IGV (sashimi plot function) to enumerate the exon-joining reads into the 5 ' boundary of exon 2 (Katz et al., 2015).

Phylogenetic analysis. MECR amino acid sequences were retrieved from NCBI (Supp. Table 4). Phylogenetic analysis was performed using Influenza Research Database (www.fludb.org) using PhyML options (Guindon and Gascuel, 2003) to generate a Newick file. FigTree v1.4.4 (http://tree.bio.ed.ac.uk/software/figtree) was used for visualization.

Statistical Analysis. Assays were performed with three to six technical replicates and represent at least three independent biological replicates. Mean and SD or SEM were calculated. When data were normalized, error was propagated to each individual experimental condition. Statistical significance was determined for pairwise comparisons with a Student's t test and for multiple comparisons an ANOVA and post hoc Dunnett's or Sidak's test. Correlation across data sets was determined via a twotailed Pearson correlation coefficient. Values from statistical analyses of siRNA screens are reported (Supp Table 3). Statistical tests were performed using Prism (v 8.4.2, GraphPad)

\section{References}

Almond, J.W. (1977). A single gene determines the host range of influenza virus. Nature 270, 617-618.

Augustin, A., Lamerz, J., Meistermann, H., Golling, S., Scheiblich, S., Hermann, J.C., Duchateau-Nguyen, G., Tzouros, M., Avila, D.W., Langen, H., et al. (2013). Quantitative chemical proteomics profiling differentiates erlotinib from gefitinib in EGFR wild-type non-small cell lung carcinoma cell lines. Mol. Cancer Ther. 12, 520-529.

Bachi, A., Braun, I.C., Rodrigues, J.P., Panté, N., Ribbeck, K., Von Kobbe, C., Kutay, U., Wilm, M., Görlich, D., CarmoFonseca, M., et al. (2000). The C-terminal domain of TAP interacts with the nuclear pore complex and promotes export of specific CTE-bearing RNA substrates. RNA 6, 136-158.

Baker, S.F., Ledwith, M.P., and Mehle, A. (2018). Differential Splicing of ANP32A in Birds Alters Its Ability to Stimulate RNA Synthesis by Restricted Influenza Polymerase. Cell Rep. 24, 2581-2588.e4.

Benjamini, Y., and Hochberg, Y. (1995). Controlling the False Discovery Rate: A Practical and Powerful Approach to Multiple Testing. J. R. Stat. Soc. Ser. B 57, 289-300.

Bock, M., Bishop, K.N., Towers, G., and Stoye, J.P. (2000). Use of a Transient Assay for Studying the Genetic Determinants of Fv1 Restriction. J. Virol. 74, 7422-7430.

Brass, A.L., Huang, I.C., Benita, Y., John, S.P., Krishnan, M.N., Feeley, E.M., Ryan, B.J., Weyer, J.L., van der Weyden, L., Fikrig, E., et al. (2009). The IFITM Proteins Mediate Cellular Resistance to Influenza A H1N1 Virus, West Nile Virus, and Dengue Virus. Cell 139, 1243-1254.

Bretz, F., and Hothorn, L.A. (2001). Testing dose-response relationships with a priori unknown, possibly nonmonotone shapes. J. Biopharm. Stat. 11, 193-207. 
bioRxiv preprint doi: https://doi.org/10.1101/2020.11.09.355982; this version posted November $10,2020$. The copyright holder for this preprint (which was not certified by peer review) is the author/funder, who has granted bioRxiv a license to display the preprint in perpetuity. It is made available under aCC-BY-NC 4.0 International license.

Busch, A., and Hertel, K.J. (2012). Evolution of SR protein and hnRNP splicing regulatory factors. Wiley Interdiscip. Rev. RNA 3, 1-12.

Bushnell, B. (2015). BBMap (version 37.75) [Software]. Available at Https://Sourceforge.Net/Projects/Bbmap/.

Carr, S.M., Carnero, E., García-Sastre, A., Brownlee, G.G., and Fodor, E. (2006). Characterization of a mitochondrialtargeting signal in the PB2 protein of influenza viruses. Virology 344, 492-508.

de Chassey, B., Aublin-Gex, A., Ruggieri, A., MeynielSchicklin, L., Pradezynski, F., Davoust, N., Chantier, T., Tafforeau, L., Mangeot, P.E., Ciancia, C., et al. (2013). The Interactomes of Influenza Virus NS1 and NS2 Proteins Identify New Host Factors and Provide Insights for ADAR1 Playing a Supportive Role in Virus Replication. PLoS Pathog. 9.

Chen, C., Han, X., Zou, X., Li, Y., Yang, L., Cao, K., Xu, J., Long, J., Liu, J., and Feng, Z. (2014). 4-Methylene-2-octyl-5oxotetrahydrofuran-3-carboxylic acid (C75), an inhibitor of fatty-acid synthase, suppressesthe mitochondrial fatty acid synthesis pathway and impairs mitochondrial function. J. Biol. Chem. 289, 17184-17194.

Chen, W.H., Lu, G., Chen, X., Zhao, X.M., and Bork, P. (2017). OGEE v2: An update of the online gene essentiality database with special focus on differentially essential genes in human cancer cell lines. Nucleic Acids Res. 45, D940D944.

Chen, Z.J., Pudas, R., Sharma, S., Smart, O.S., Juffer, A.H., Hiltunen, J.K., Wierenga, R.K., and Haapalainen, A.M. (2008). Structural Enzymological Studies of 2-Enoyl Thioester Reductase of the Human Mitochondrial FAS II Pathway: New Insights into Its Substrate Recognition Properties. J. Mol. Biol. 379, 830-844.

Daugherty, M.D., and Malik, H.S. (2012). Rules of engagement: Molecular insights from host-virus arms races. Annu. Rev. Genet. 46, 677-700.

Dawood, F.S., Jain, S., Finelli, L., Shaw, M.W., Lindstrom, S., Garten, R.J., Gubareva, L. V., Xu, X., Bridges, C.B., and Uyeki, T.M. (2009). Emergence of a novel swine-origin influenza A (H1N1) virus in humans. N. Engl. J. Med. 360, 2605-2615.

Dawson, A.R., Wilson, G.M., Coon, J.J., and Mehle, A. (2020). Post-Translation Regulation of Influenza Virus Replication. Annu. Rev. Virol. 7.

Delaleau, M., and Borden, K. (2015). Multiple Export Mechanisms for mRNAs. Cells 4, 452-473.

Dhir, A., Dhir, S., Borowski, L.S., Jimenez, L., Teitell, M., Rötig, A., Crow, Y.J., Rice, G.I., Duffy, D., Tamby, C., et al. (2018). Mitochondrial double-stranded RNA triggers antiviral signalling in humans. Nature 560, 238-242.

Engelhardt, O.G., Smith, M., and Fodor, E. (2005). Association of the Influenza A Virus RNA-Dependent RNA Polymerase with Cellular RNA Polymerase II. J. Virol. 79, 5812-5818.

Feng, D., Witkowski, A., and Smith, S. (2009). Downregulation of mitochondrial acyl carrier protein in mammalian cells compromises protein lipoylation and respiratory complex I and results in cell death. J. Biol. Chem. 284, 11436-11445.

Fodor, E., and te Velthuis, A.J.W. (2019). Structure and Function of the Influenza Virus Transcription and Replication Machinery. Cold Spring Harb. Perspect. Med. a038398.
Gabler, S., Schütt, H., Groitl, P., Wolf, H., Shenk, T., and Dobner, T. (1998). E1B 55-Kilodalton-Associated Protein: a Cellular Protein with RNA-Binding Activity Implicated in Nucleocytoplasmic Transport of Adenovirus and Cellular mRNAs. J. Virol. 72, 7960-7971.

Gabriel, G., Klingel, K., Otte, A., Thiele, S., Hudjetz, B., Arman-Kalcek, G., Sauter, M., Shmidt, T., Rother, F., Baumgarte, S., et al. (2011). Differential use of importin-a isoforms governs cell tropism and host adaptation of influenza virus. Nat. Commun. 2, 156.

Gansner, E.R., and North, S.C. (2000). Open graph visualization system and its applications to software engineering. Softw. - Pract. Exp. 30, 1203-1233.

García, M.A., Gil, J., Ventoso, I., Guerra, S., Domingo, E., Rivas, C., and Esteban, M. (2006). Impact of Protein Kinase PKR in Cell Biology: from Antiviral to Antiproliferative Action. Microbiol. Mol. Biol. Rev. 70, 1032-1060.

Garten, R., Blanton, L., Elal, A.I.A., Alabi, N., Barnes, J., Biggerstaff, M., Brammer, L., Budd, A.P., Burns, E., Cummings, C.N., et al. (2018). Update: Influenza Activity in the United States During the 2017-18 Season and Composition of the 2018-19 Influenza Vaccine. MMWR. Morb. Mortal. Wkly. Rep. 67, 634-642.

Geuens, T., Bouhy, D., and Timmerman, V. (2016). The hnRNP family: insights into their role in health and disease. Hum. Genet. 135, 851-867.

Graef, K.M., Vreede, F.T., Lau, Y.-F., McCall, A.W., Carr, S.M., Subbarao, K., and Fodor, E. (2010). The PB2 Subunit of the Influenza Virus RNA Polymerase Affects Virulence by Interacting with the Mitochondrial Antiviral Signaling Protein and Inhibiting Expression of Beta Interferon. J. Virol. 84, 8433-8445.

Guindon, S., and Gascuel, O. (2003). A Simple, Fast, and Accurate Algorithm to Estimate Large Phylogenies by Maximum Likelihood. Syst. Biol. 52, 696-704.

Gurunathan, G., Yu, Z., Coulombe, Y., Masson, J.Y., and Richard, S. (2015). Arginine methylation of hnRNPUL1 regulates interaction with NBS1 and recruitment to sites of DNA damage. Sci. Rep. 5, 1-9.

Hao, L., Sakurai, A., Watanabe, T., Sorensen, E., Nidom, C.A., Newton, M.A., Ahlquist, P., and Kawaoka, Y. (2008). Drosophila RNAi screen identifies host genes important for influenza virus replication. Nature 454, 890-893.

Hou, F., Sun, L., Zheng, H., Skaug, B., Jiang, Q.X., and Chen, Z.J. (2011). MAVS forms functional prion-like aggregates to activate and propagate antiviral innate immune response. Cell 146, 448-461.

Hsiau, T., Conant, D., Rossi, N., Maures, T., Waite, K., Yang, J., Joshi, S., Kelso, R., Holden, K., Enzmann, B., et al. (2018). Inference of CRISPR Edits from Sanger Trace Data. BioRxiv 251082.

Karlas, A., MacHuy, N., Shin, Y., Pleissner, K.P., Artarini, A., Heuer, D., Becker, D., Khalil, H., Ogilvie, L.A., Hess, S., et al. (2010). Genome-wide RNAi screen identifies human host factors crucial for influenza virus replication. Nature 463 , 818-822.

Karlsson, E.A., Meliopoulos, V.A., Savage, C., Livingston, B., Mehle, A., and Schultz-Cherry, S. (2015). Visualizing realtime influenza virus infection, transmission and protection in ferrets. Nat. Commun. 6, 6378 . 
bioRxiv preprint doi: https://doi.org/10.1101/2020.11.09.355982; this version posted November $10,2020$. The copyright holder for this preprint (which was not certified by peer review) is the author/funder, who has granted bioRxiv a license to display the preprint in perpetuity. It is made available under aCC-BY-NC 4.0 International license.

Katz, Y., Wang, E.T., Silterra, J., Schwartz, S., Wong, B., Thorvaldsdóttir, H., Robinson, J.T., Mesirov, J.P., Airoldi, E.M., and Burge, C.B. (2015). Quantitative visualization of alternative exon expression from RNA-seq data. Bioinformatics 31, 2400-2402.

Kawaguchi, A., and Nagata, K. (2007). De novo replication of the influenza virus RNA genome is regulated by DNA replicative helicase, MCM. EMBO J. 26, 4566-4575.

Kim, D., Paggi, J.M., Park, C., Bennett, C., and Salzberg, S.L. (2019). Graph-based genome alignment and genotyping with HISAT2 and HISAT-genotype. Nat. Biotechnol. 37, 907915.

Kim, D.G., Yoo, J.C., Kim, E., Lee, Y.S., Yarishkin, O. V., Lee, D.Y., Lee, K.H., Hong, S.G., Hwang, E.M., and Park, J.Y. (2014). A novel cytosolic isoform of mitochondrial trans2-enoyl-CoA reductase enhances peroxisome proliferatoractivated receptor a activity. Endocrinol. Metab. 29, 185-194. Kirui, J., Bucci, M.D., Poole, D.S., and Mehle, A. (2014). Conserved Features of the PB2 627 Domain Impact Influenza Virus Polymerase Function and Replication. J. Virol. 88, 5977-5986.

König, R., Stertz, S., Zhou, Y., Inoue, A., Hoffmann, H.H., Bhattacharyya, S., Alamares, J.G., Tscherne, D.M., Ortigoza, M.B., Liang, Y., et al. (2010). Human host factors required for influenza virus replication. Nature 463, 813-817.

Larson, G.P., Tran, V., Yú, S., Caì, Y., Higgins, C.A., Smith, D.M., Baker, S.F., Radoshitzky, S.R., Kuhn, J.H., and Mehle, A. (2019). EPS8 Facilitates Uncoating of Influenza A Virus. Cell Rep. 29, 2175-2183.e4.

Long, J.C.D., and Fodor, E. (2016). The PB2 Subunit of the Influenza A Virus RNA Polymerase Is Imported into the Mitochondrial Matrix. J. Virol. 90, 8729-8738.

Long, J.S., Giotis, E.S., Moncorgé, O., Frise, R., Mistry, B., James, J., Morisson, M., Iqbal, M., Vignal, A., Skinner, M.A., et al. (2016). Species difference in ANP32A underlies influenza A virus polymerase host restriction. Nature 529, 101-104.

Long, J.S., Idoko-Akoh, A., Mistry, B., Goldhill, D., Staller, E., Schreyer, J., Ross, C., Goodbourn, S., Shelton, H., Skinner, M.A., et al. (2019). Species specific differences in use of ANP32 proteins by influenza A virus. Elife 8.

Marceau, C.D., Puschnik, A.S., Majzoub, K., Ooi, Y.S., Brewer, S.M., Fuchs, G., Swaminathan, K., Mata, M.A., Elias, J.E., Sarnow, P., et al. (2016). Genetic dissection of Flaviviridae host factors through genome-scale CRISPR screens. Nature 535, 159-163.

Mehle, A., and Doudna, J.A. (2008). An Inhibitory Activity in Human Cells Restricts the Function of an Avian-like Influenza Virus Polymerase. Cell Host Microbe 4, 111-122.

Mehle, A., and Doudna, J.A. (2009). Adaptive strategies of the influenza virus polymerase for replication in humans. Proc. Natl. Acad. Sci. U. S. A. 106, 21312-21316.

Meistermann, H., Gao, J., Golling, S., Lamerz, J., Le Pogam, S., Tzouros, M., Sankabathula, S., Gruenbaum, L., Nájera, I., Langen, H., et al. (2014). A novel immuno-competitive capture mass spectrometry strategy for protein-protein interaction profiling reveals that LATS kinases regulate HCV replication through NS5A phosphorylation. Mol. Cell.

Proteomics 13, 3040-3048.

Meyer, F. (2016). Viral interactions with components of the splicing machinery. In Progress in Molecular Biology and Translational Science, pp. 241-268.
Nair, R.R., Kerätär, J.M., Autio, K.J., Masud, A.J., Finnilä, M.A.J., Autio-Harmainen, H.I., Miinalainen, I.J., Nieminen, P.A., Hiltunen, J.K., and Kastaniotis, A.J. (2017). Genetic modifications of Mecr reveal a role for mitochondrial 2-enoylCoA/ACP reductase in placental development in mice. Hum. Mol. Genet. 26, 2104-2117.

Neumann, G., Hughes, M.T., and Kawaoka, Y. (2000). Influenza A virus NS2 protein mediates VRNP nuclear export through NES-independent interaction with hCRM1. EMBO J. 19, 6751-6758.

Neumann, G., Fujii, K., Kino, Y., and Kawaoka, Y. (2005). An improved reverse genetics system for influenza $A$ virus generation and its implications for vaccine production. Proc. Natl. Acad. Sci. U. S. A. 102, 16825-16829.

Nowinski, S.M., Van Vranken, J.G., Dove, K.K., and Rutter, J. (2018). Impact of Mitochondrial Fatty Acid Synthesis on Mitochondrial Biogenesis. Curr. Biol. 28, R1212-R1219.

Nowinski, S.M., Solmonson, A., Rusin, S.F., Maschek, J.A., Bensard, C.L., Fogarty, S., Jeong, M.-Y., Lettlova, S., Berg, J.A., Morgan, J.T., et al. (2020). Mitochondrial fatty acid synthesis coordinates oxidative metabolism in mammalian mitochondria. Elife 9.

Ozdilek, B.A., Thompson, V.F., Ahmed, N.S., White, C.I., Batey, R.T., and Schwartz, J.C. (2017). Intrinsically disordered RGG/RG domains mediate degenerate specificity in RNA binding. Nucleic Acids Res. 45, 7984-7996.

Peacock, T.P., Sheppard, C.M., Staller, E., and Barclay, W.S. (2019). Host Determinants of Influenza RNA Synthesis. Annu. Rev. Virol. 6, 215-233.

Polo, S.E., Blackford, A.N., Chapman, J.R., Baskcomb, L., Gravel, S., Rusch, A., Thomas, A., Blundred, R., Smith, P., Kzhyshkowska, J., et al. (2012). Regulation of DNA-End Resection by hnRNPU-like Proteins Promotes DNA DoubleStrand Break Signaling and Repair. Mol. Cell 45, 505-516.

R Core Team (2019). R: A language and environment for statistical computing. R Found. Stat. Comput. Vienna, Austria.

Rahim, M.M.A., Parsons, B.D., Price, E.L., Slaine, P.D., Chilvers, B.L., Seaton, G.S., Wight, A., Medina-Luna, D., Dey, S., Grandy, S.L., et al. (2020). Defective Influenza A Virus RNA Products Mediate MAVS-Dependent Upregulation of Human Leukocyte Antigen Class I Proteins. J. Virol. 94.

Raudvere, U., Kolberg, L., Kuzmin, I., Arak, T., Adler, P., Peterson, H., and Vilo, J. (2019). G:Profiler: A web server for functional enrichment analysis and conversions of gene lists (2019 update). Nucleic Acids Res. 47, W191-W198.

Read, E.K.C., and Digard, P. (2010). Individual influenza A virus mRNAs show differential dependence on cellular NXF1/TAP for their nuclear export. J. Gen. Virol. 91, 12901301.

Refolo, G., Vescovo, T., Piacentini, M., Fimia, G.M., and Ciccosanti, F. (2020). Mitochondrial Interactome: A Focus on Antiviral Signaling Pathways. Front. Cell Dev. Biol. 8.

Rehwinkel, J., and Gack, M.U. (2020). RIG-I-like receptors: their regulation and roles in RNA sensing. Nat. Rev. Immunol.

Reilly, P.T., Afzal, S., Gorrini, C., Lui, K., Bukhman, Y. V., Wakeham, A., Haight, J., Ling, T.W., Cheung, C.C., Elia, A.J., et al. (2011). Acidic nuclear phosphoprotein $32 \mathrm{kDa}$ (ANP32)B-deficient mouse reveals a hierarchy of ANP32 importance in mammalian development. Proc. Natl. Acad. Sci. U. S. A. 108, 10243-10248. 
Rialdi, A., Hultquist, J., Jimenez-Morales, D., Peralta, Z., Campisi, L., Fenouil, R., Moshkina, N., Wang, Z.Z., Laffleur, B., Kaake, R.M., et al. (2017). The RNA Exosome Syncs IAV-RNAPII Transcription to Promote Viral Ribogenesis and Infectivity. Cell 169, 679-692.e14.

Ritchie, M.E., Phipson, B., Wu, D., Hu, Y., Law, C.W., Shi, W., and Smyth, G.K. (2015). Limma powers differential expression analyses for RNA-sequencing and microarray studies. Nucleic Acids Res. 43, e47.

Rupp, J.C., Locatelli, M., Grieser, A., Ramos, A., Campbell, P.J., Yi, H., Steel, J., Burkhead, J.L., and Bortz, E. (2017). Host Cell Copper Transporters CTR1 and ATP7A are important for Influenza A virus replication. Virol. J. 14, 1-12. Dos Santos Afonso, E., Escriou, N., Leclercq, I., Van Der Werf, S., and Naffakh, N. (2005). The generation of recombinant influenza A viruses expressing a $\mathrm{PB} 2$ fusion protein requires the conservation of a packaging signal overlapping the coding and noncoding regions at the $5^{\prime}$ end of the PB2 segment. Virology 341, 34-46.

Satterly, N., Tsai, P.L., Van Deursen, J., Nussenzveig, D.R., Wang, Y., Faria, P.A., Levay, A., Levy, D.E., and Fontoura, B.M.A. (2007). Influenza virus targets the mRNA export machinery and the nuclear pore complex. Proc. Natl. Acad. Sci. U. S. A. 104, 1853-1858.

Shapira, S.D., Gat-Viks, I., Shum, B.O.V., Dricot, A., de Grace, M.M., Wu, L., Gupta, P.B., Hao, T., Silver, S.J., Root, D.E., et al. (2009). A Physical and Regulatory Map of HostInfluenza Interactions Reveals Pathways in H1N1 Infection. Cell 139, 1255-1267.

Smyth, G.K. (2004). Linear models and empirical bayes methods for assessing differential expression in microarray experiments. Stat. Appl. Genet. Mol. Biol. 3.

Sorouri, M., Chang, T., Jesudhasan, P., Pinkham, C., Elde, N., and Hancks, D. (2020). MISTR: A conserved Mitochondrial STress Response network revealed by signatures of evolutionary conflict. BioRxiv.

Stewart, W.H., and Ruberg, S.J. (2000). Detecting dose response with contrasts. Stat. Med. 19, 913-921.

Subbarao, E.K., London, W., and Murphy, B.R. (1993). A single amino acid in the PB2 gene of influenza $A$ virus is a determinant of host range. J. Virol. 67, 1761-1764.

Sugiyama, K., Kawaguchi, A., Okuwaki, M., and Nagata, K. (2015). PP32 and APRIL are host cell-derived regulators of influenza virus RNA synthesis from cRNA. Elife 4.

Szklarczyk, D., Franceschini, A., Wyder, S., Forslund, K., Heller, D., Huerta-Cepas, J., Simonovic, M., Roth, A.,

Santos, A., Tsafou, K.P., et al. (2015). STRING v10: Proteinprotein interaction networks, integrated over the tree of life. Nucleic Acids Res. 43, D447-D452.

Tran, V., Moser, L.A., Poole, D.S., and Mehle, A. (2013). Highly Sensitive Real-Time In Vivo Imaging of an Influenza Reporter Virus Reveals Dynamics of Replication and Spread. J. Virol. 87, 13321-13329.

Tran, V., Ledwith, M.P., Thamamongood, T., Higgins, C.A., Tripathi, S., Chang, M.W., Benner, C., García-Sastre, A., Schwemmle, M., Boon, A.C.M., et al. (2020). Influenza virus repurposes the antiviral protein IFIT2 to promote translation of viral mRNAs. Nat. Microbiol. 1-14.
Tripathi, S., Pohl, M.O., Zhou, Y., Rodriguez-Frandsen, A., Wang, G., Stein, D.A., Moulton, H.M., Dejesus, P., Che, J., Mulder, L.C.F., et al. (2015). Meta- and Orthogonal Integration of Influenza "oMICs" Data Defines a Role for UBR4 in Virus Budding. Cell Host Microbe 18, 723-735.

Tsai, P.L., Chiou, N.T., Kuss, S., García-Sastre, A., Lynch, K.W., and Fontoura, B.M.A. (2013). Cellular RNA Binding Proteins NS1-BP and hnRNP K Regulate Influenza A Virus RNA Splicing. PLoS Pathog. 9.

Van Valen, L. (1976). The Red Queen lives. Nature 260, 575.

Varmuza, K., and Filzmoser, P. (2016). Introduction to Multivariate Statistical Analysis in Chemometrics (CRC Press).

Wandzik, J.M., Kouba, T., and Cusack, S. (2020). Structure and Function of Influenza Polymerase. Cold Spring Harb. Perspect. Med. a038372.

Westfall, P.H., and Young, S.S. (1993). Resampling-based multiple testing: Examples and methods for $p$-value adjustment (Wiley).

Yeger-Lotem, E., Riva, L., Su, L.J., Gitler, A.D., Cashikar, A.G., King, O.D., Auluck, P.K., Geddie, M.L., Valastyan, J.S., Karger, D.R., et al. (2009). Bridging high-throughput genetic and transcriptional data reveals cellular responses to alphasynuclein toxicity. Nat. Genet. 41, 316-323.

\section{Acknowledgements}

We thank members of the Mehle lab for critical reading of the manuscript. We thank Craig McCormick (Dalhousie University) for sharing reagents. This work was supported by NIH/NIAID R01Al125271 and the Greater Milwaukee Foundation Shaw Scientist Award to AM, R21Al125897 to AM and AG, a Roche Postdoctoral Fellowship RPF-353 and the NIAID T32Al55397 to SFB, T32LM012413 to AB, and an NSF GRFP DGE-1747503 to MPL. AM is a Burroughs Wellcome Fund Investigator in the Pathogenesis of Infectious Disease.

\section{Author contributions}

Conceptualization: SFB, HM, MT, AB, AG, AA, $\mathrm{HJ}, \mathrm{AM}$

Methodology: SFB, HM, MT, AB, SG, AA

Formal Analysis: SFB, HM, MT, AB, JSP, MPL, AG, AA, AM, Investigation: SFB, HM, MT, AB, MPL

Writing - Original Draft: SFB, AB, AG, AM

Writing - Review \& Editing: all

Visualization: SFB, AB

Funding Acquisition: SFB, MPL, AM, HJ

Supervision: $H M, A G, A A, H J, A M$

\section{Declaration of interests}

The authors declare no competing interests. 